\title{
InVentario florístico de la Reserva de la Biosfera SieRra del Abra Tanchipa, San LUIS Potosí, MÉXICO
}

\section{Floristic inVentory of the Biosphere Reserve SierRa del Abra Tanchipa, San LUIS Potosí, MeXICO}

\author{
José Arturo De-Nova ${ }^{1, *}$, Ricardo González-Trujillo ${ }^{2}$, Pedro Castillo-Lara ${ }^{1}$, Javier Fortanelli-Martínez ${ }^{1}$, \\ ARTURo Mora-Olivo ${ }^{3}$ y María Magdalena Salinas-RodríGUez ${ }^{1}$
}

${ }^{1}$ Instituto de Investigaciones de Zonas Desérticas, Universidad Autónoma de San Luis Potosí, San Luis Potosí, México. ${ }^{2}$ Centro de Investigación para los Recursos Naturales, Chihuahua, México.

${ }^{3}$ Instituto de Ecología Aplicada, Universidad Autónoma de Tamaulipas, Cd. Victoria, Tamaulipas, México.

*Autor para la correspondencia: arturo.denova@gmail.com

\section{Resumen}

Antecedentes: Los bosques tropicales estacionalmente secos de México incluyen más de tres mil especies de plantas vasculares. La Reserva de la Biosfera Sierra del Abra Tanchipa representa un eslabón para este tipo de bosques en la región noreste de México.

Pregunta: ¿Cuál es la composición y riqueza de plantas vasculares en la Reserva de la Biosfera Sierra del Abra Tanchipa, sus formas de crecimiento, endemismo, usos y estado de conservación?

Sitio de estudio y fechas: Reserva de la Biosfera Sierra del Abra Tanchipa, San Luis Potosí, México, de diciembre de 2012 a enero de 2018.

Métodos: Se realizaron recolectas botánicas en un gradiente de 100 a $700 \mathrm{~m}$ snm. Se tomaron datos sobre el hábitat, fenología, forma de crecimiento, nombres comunes y usos.

Resultados: Se registraron 89 familias, 305 géneros y 427 especies de plantas vasculares. Las familias con más especies fueron Fabaceae, Orchidaceae, Poaceae, Asteraceae y Euphorbiaceae. El área incluye 46 especies endémicas de México y siete especies en alguna categoría de riesgo según la NOM-059-SEMARNAT-2010. Entre las 116 especies útiles y/o tóxicas destacan las ornamentales, para construcción, comestibles y medicinales.

Conclusiones: La riqueza de especies en el área es similar a la registrada en otras regiones de bosques tropicales estacionalmente secos en México. El buen estado de conservación de la reserva, su conexión con otros fragmentos de bosques hacia el norte, así como la presencia de especies endémicas y en alguna categoría de riesgo, resaltan su importancia para la conservación de estos ecosistemas en México.

Palabras clave: Bosque tropical caducifolio, endemismo, especies en riesgo, plantas útiles, vegetación.

\section{Abstract}

Background: Seasonally dry tropical forests of Mexico include over three thousand species of vascular plants. The Sierra del Abra Tanchipa Biosphere Reserve represents a link for this kind of forests in northern Mexico.

Question: What is the composition and richness of vascular plants in the Sierra del Abra Tanchipa Biosphere Reserve, its growth forms, endemism, uses, and conservation status?

Study site and research period: Sierra del Abra Tanchipa Biosphere Reserve, San Luis Potosi, Mexico, between December 2012 to January 2018.

Methods: Botanical collections were carried out in a gradient from 100 to $700 \mathrm{~m}$ asl. Data for habitat, phenology, growth form, common names and uses were recorded.

Results: 89 families, 305 genera and 427 species of vascular plants were recorded. The richest families were Fabaceae, Orchidaceae, Poaceae, Asteraceae, and Euphorbiaceae. The area includes 46 endemic species to Mexico, and eight in some risk category according to NOM-059SEMARNAT-2010. Among the 116 useful and/or toxic species stand out those used as ornamental, for construction purposes, as food and medicine.

Conclusions: Species richness in the area is similar to those of other regions with seasonally dry tropical forests of Mexico. The good state of conservation within the reserve, its connection with ecosystems to the North, as well as the presence of endemic species and in some risk category, highlight its importance for conservation of these ecosystems in Mexico.

Keywords: Deciduous tropical forest, endemism, endangered species, useful plants. 
La flora de México es considerada como una de las más ricas del mundo con 23,314 especies nativas y 11,600 especies endémicas, de las cuales al menos 3,225 habitan en bosques tropicales estacionalmente secos (Rzedowski \& Calderón de Rzedowski 2013, Villaseñor 2016). Este ecosistema es uno de los más amenazados en el Neotrópico en donde su distribución original se ha reducido drásticamente (Pennington et al. 2000, Pennington et al. 2004, Dick \& Wright 2005, Pennington et al. 2009, De-Nova et al. 2012, Pizano et al. 2014). En México ocupa 18,739,273.92 hectáreas que corresponde al $9.49 \%$ del territorio nacional (Trejo \& Dirzo 2002, INEGI 2013). Los bosques tropicales estacionalmente secos incluyen una variedad de asociaciones vegetales que van desde bosques tropicales altos, relativamente húmedos, hasta asociaciones de menor altura similares a matorrales y ricos en suculentas en regiones más secas. Se distribuyen principalmente en zonas de clima cálido con temperaturas promedio de hasta $30{ }^{\circ} \mathrm{C}$ y precipitaciones menores a los 1,200 mm anuales (Trejo-Vázquez 1999); aunque otros autores los mencionan para precipitaciones menores a los 1,800 $\mathrm{mm}$ anuales, pero con una estación de cinco a seis meses en donde se reciben menos de $100 \mathrm{~mm}$ (Pennington et al. 2009, De-Nova et al. 2012), cuando la mayoría de sus elementos arbóreos pierden las hojas. Su principal distribución en México es en la vertiente del Océano Pacífico, desde Sonora hasta Centroamérica, y de manera discontinua en la vertiente del Atlántico, desde Tamaulipas hasta la Península de Yucatán; principalmente desde 0 hasta 1,500 m snm. (Rzedowski 1978).

En México, los bosques tropicales estacionalmente secos han sido clasificados de diferentes maneras, como son: bosque tropical deciduo, bosque tropical caducifolio y subcaducifolio, selva baja caducifolia y subcaducifolia, selva mediana caducifolia y subcaducifolia (Miranda \& Hernández-X 1963, Rzedowski 1978, INEGI 2009, Challenger \& Soberón 2008). Para el estado de San Luis Potosí se reconoce como bosque tropical deciduo o caducifolio, o como selva baja caducifolia, y sus elementos más importantes tienen alturas de 8 a $15 \mathrm{~m}$ y un porte característico de paraguas, ramificados cerca de la base y con una copa ensanchada (Rzedowski 1965, Rzedowski 1978, Puig 1991). Esta vegetación se distribuye en los declives orientales inferiores de la Sierra Madre Oriental entre los municipios de San Martín Chalchicuautla, Ciudad Valles, Tamasopo y El Naranjo, a elevaciones desde los 50 a los $800 \mathrm{~m}$ snm, principalmente en sustratos sedimentarios con suelos someros sobre la roca madre.

Un área importante para la conservación de estos ecosistemas estacionalmente secos en el estado de San Luis Potosí es la Reserva de la Biosfera Sierra del Abra Tanchipa (RBSAT), en donde el nivel de conservación de sus comunidades vegetales es sobresaliente, con niveles de disturbio casi nulos (De-Nova et al. 2018, Rubio-Méndez et al. 2018). Los primeros estudios sobre la flora de esta área natural protegida fueron realizados por Mora-Olivo et al. (1992) como parte del estudio técnico justificativo para la declaratoria de la reserva. En ese entonces se registraron 231 especies de plantas vasculares distribuidas en variantes de bosques tropicales estacionales, denominadas selva baja y mediana caducifolia y subperennifolia, encinar tropical, palmar y vegetación secundaria. Sin embargo, aunque el muestreo incluyó un área mayor a la que actualmente representa el área núcleo de la reserva, fue limitado debido al tiempo con el que se contó para realizarlo.

En este trabajo presentamos los resultados de seis años de exploración botánica en la RBSAT, en donde además se incluyen recolectas históricas para la región y algunas especies observadas registradas en el portal NaturaLista de CONABIO. El objetivo de este trabajo es contribuir al conocimiento florístico de áreas de bosques tropicales estacionalmente secos con alto grado de conservación. Se presenta una lista actualizada y anotada de las plantas vasculares para la RBSAT que funcionará como insumo para generar estrategias de conservación de estas áreas casi intactas.

\section{Materiales y métodos}

Zona de estudio. La RBSAT se localiza en los municipios de Ciudad Valles y Tamuín al este del estado de San Luis Potosí, comprende una superficie total de 21,464 hectáreas y se ubica entre las coordenadas $22^{\circ} 05^{\prime} 00^{\prime \prime}$ ' y $22^{\circ} 24^{\prime} 22^{\prime \prime}$ ' de latitud norte y $98^{\circ} 52^{\prime} 46^{\prime}$ ' y $99^{\circ} 01^{\prime}$ '00', de longitud oeste (DOF 1994, CONANP 2014). Es una sierra formada en su totalidad de rocas sedimentarias marinas predominantemente calcáreas de permeabilidad alta del Cretácico Inferior (INEGI 1988), con geoformas como sierras, laderas, cañadas y lomeríos en donde se desarrollaron importantes procesos de karstificación como sótanos, cavernas y dolinas, así como mogotes rocosos donde habitan especies rupícolas. Es una sierra con elevaciones de entre 100 y $700 \mathrm{~m}$ snm, con una forma alargada que va de norte a sur. Su flanco oriental es escarpado mientras que su flanco occidental desciende suavemente en varios pisos altitudinales y pertenece a una porción de la subprovincia fisiográfica Gran Sierra Plegada, pero con muchas características propias de la subprovincia Carso Huasteco (Cervantes-Zamora et al. 1990). En el área abundan los suelos arcillosos someros de color rojizo que no rebasan los $40 \mathrm{~cm}$ de profundidad y son derivados de litosoles (INEGI 2013). Pertenece a la Región Hidrológica del Río Pánuco, con un tipo de cuenca exorreica y drenaje angulado, en la RBSAT no hay ríos ni arroyos permanentes, solo escurrimientos superficiales que se hacen manifiestos en la época de lluvias y huracanes. Así mismo, el proceso de karstificación de la sierra ha creado una red de cavernas por donde hay escurrimientos subterráneos (CONANP 2014). Según la clasificación de Köppen modificado por García (1981) el clima del área es cálido subhúmedo Aw(2), el más húmedo de los subhúmedos, que se caracteriza por presentar temperaturas medias anuales mayores a los $22^{\circ} \mathrm{C}$ y temperaturas del mes más frío menor de $18{ }^{\circ} \mathrm{C}$ así como precipitaciones medias anuales entre 1,200 y $1,500 \mathrm{~mm}$, con 0 a $60 \mathrm{~mm}$ en el mes más seco, lluvias en verano con índice $\mathrm{P} / \mathrm{T}$ mayor de 55.3 y un porcentaje de lluvia invernal del 5 al $10.2 \%$ del total anual. En el área predomina lo que se denomina bosque tropical caducifolio o selva baja caducifolia, con un promedio de altura de $12 \mathrm{~m}$, en donde la mayoría de sus especies arbóreas y/o arbustivas pierden sus hojas 
durante la temporada seca y destacan los elementos de hojas compuestas (Puig 1991).

Trabajo de campo y gabinete. Se realizaron exploraciones y recolectas sistematizadas de oeste a este en seis transectos de recolecta en las fracciones norte, centro y sur de la RBSAT (Figura 1), durante todas las estaciones del año de diciembre del 2012 a enero del 2018. Se entrevistó a personas de los poblados y comunidades cercanas con el fin de obtener nombres de los puntos de referencia para precisar las localidades. Estos recorridos se realizaron con ayuda de guías locales que además nos proporcionaron nombres comunes y usos para las especies registradas. Para cada recolecta se tomaron datos referentes al hábitat, suelo, clima, georreferencia, elevación, fenología, forma de crecimiento, grado de conservación de la especie en el punto de recolecta, usos y amenazas. Con el fin de ilustrar la apariencia estructural de cada especie se asignaron formas de crecimiento generales de acuerdo con conceptos definidos en el glosario botánico de Font Quer (1953). Además, se indicó el tipo de nutrición para los casos especiales como parásitas y saprófitas. El procesamiento e identificación de los ejemplares botánicos se realizó en el herbario SLPM (Herbario Isidro Palacios, Instituto de Investigaciones de Zonas Desérticas, Universidad Autónoma de San Luis Potosí) en donde se depositó el primer duplicado de cada número de recolecta. El resto de los duplicados se distribuyeron a los herbarios HGOM (Centro de Investigaciones Biológicas, Universidad Autónoma del Estado de Hidalgo), IBUG (Centro de Investigaciones Biológicas, Universidad Autónoma del Estado de Hidalgo), QMEX (Herbario 'Dr. Jerzy Rzedowski', de la Facultad de Ciencias Naturales, Universidad Autónoma de Querétaro) y UAT (Instituto de Ecología Aplicada, Universidad Autónoma de Tamaulipas). Algunas de las especies se registraron de manera fotográfica y fueron incluidas dentro del portal Naturalista (CONABIO 2019). Adicionalmente se consultaron especímenes históricos para la región en colecciones de los herbarios SLPM y UAT.

La estandarización de la nomenclatura se realizó con el paquete Taxonstand v.2.1 (Cayuela et al. 2017) para $\mathrm{R}(\mathrm{R}$ Core Development Team 2015) la cual se basa en ThePlantList (2010). La clasificación se realizó de acuerdo con el sistema del APG (2016) para angiospermas; para los helechos y afines, y para gimnospermas se consideraron los sistemas de Christenhusz et al. (2011a, 2011b). Se realizó una revisión exhaustiva del marco legal para conocer las especies incluidas en alguna categoría de conservación nacional en la NOM-059-ECOL-2010 (SEMARNAT 2010), así como de las especies endémicas de México de acuerdo con Villaseñor (2016).

Con el fin de comparar la riqueza florística de la RBSAT $\mathrm{y}$ otras áreas con bosques tropicales estacionalmente secos
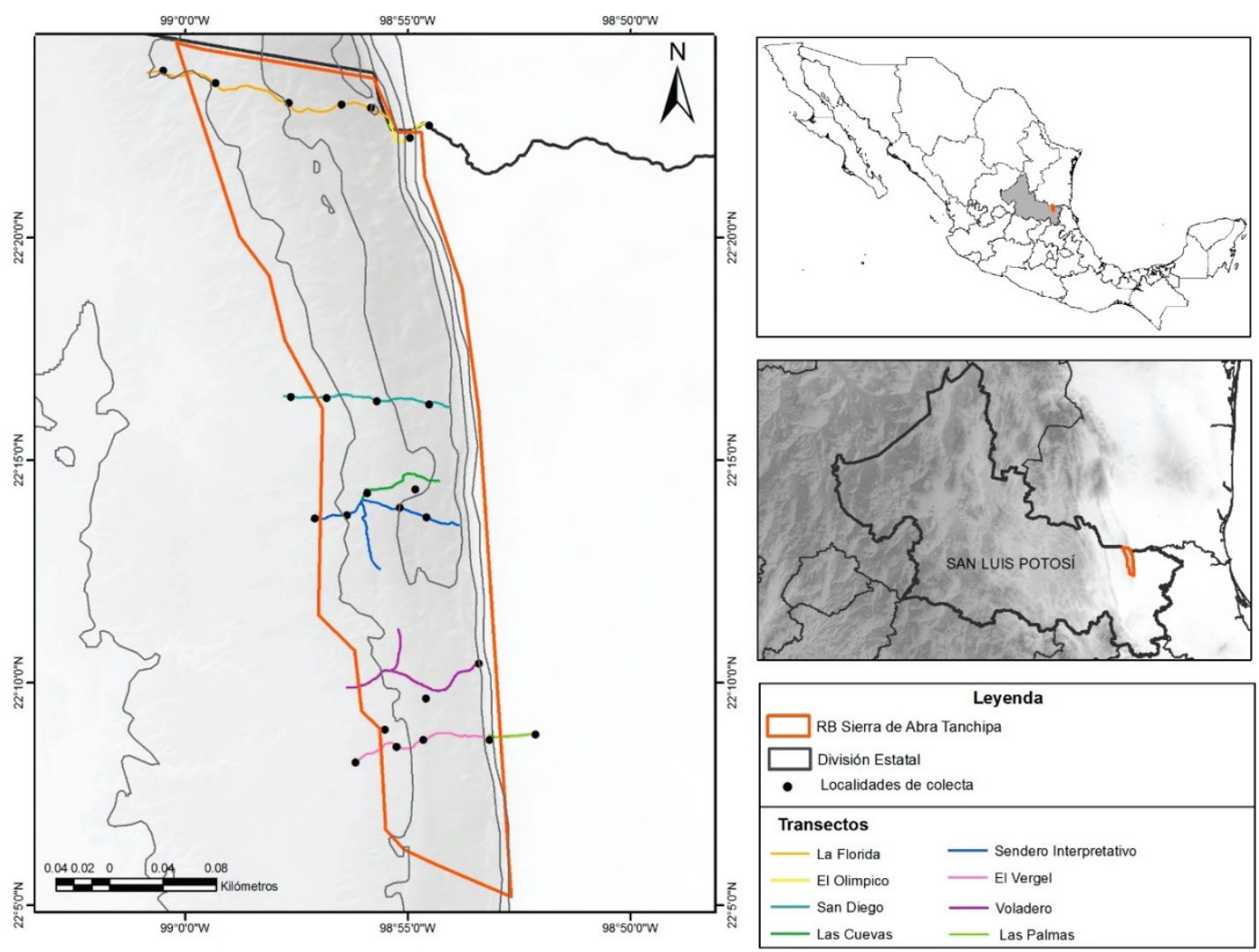

Figura 1. Ubicación y trayectos de muestreo para el conocimiento de la flora vascular en la RBSAT, San Luis Potosí, México. 
en México previamente estudiadas, se calculó el índice de biodiversidad taxonómica de acuerdo con Squeo et al. (1998) definido como el número de especies entre el logaritmo natural del área en $\mathrm{km}^{2}(I B=S / \ln A)$.

\section{Resultados}

Trabajo de campo. En total se realizaron 707 recolectas durante 61 días en campañas de tres días en promedio y que incluyeron todas las estaciones del año, los ejemplares pueden ser consultados en la base de datos del herbario Isidro Palacios (SLPM 2019). Las observaciones registradas de manera fotográfica forman parte del proyecto 2301 de Naturalista (CONABIO 2019, De-Nova et al. 2019). Los especímenes históricos incluyen cerca de 50 ejemplares recolectados desde 1954 hasta 1999.

Riqueza taxonómica. Se registró un total de 427 especies de plantas vasculares pertenecientes a 305 géneros y 89 familias (Apéndice 1), 409 con respaldo de ejemplar de herbario y 18 observados y registrados en Naturalista. Las eudicotiledóneas son el grupo taxonómico mejor representado con 308 especies $(72.13 \%)$, seguidas de las monocotiledóneas con 98 especies $(22.95 \%)$, y las restantes se dividen en magnolídeas con 13 especies, gimnospermas con dos especies y los helechos y afines con 11 especies (Tabla 1). Las familias más diversas fueron Fabaceae (28 géneros, 40 especies), Orchidaceae (23 géneros, 28 especies), Poaceae (15 géneros, 21 especies), Asteraceae (18 géneros, 20 especies) y Euphorbiaceae (nueve géneros, 20 especies) (Tabla 2). Los géneros más diversos fueron Ipomea, Peperomia y Tillandsia con siete especies cada uno, seguidos de Euphorbia y Solanum con seis especies cada uno. En las figuras 2, 3 y 4 se muestran algunas especies de la RBSAT. Respecto a las formas de crecimiento se cuentan 214 especies de hierbas, 78 árboles, 59 arbustos, 47 trepadoras, y 29 epífitas (Tabla 3). Resalta la presencia de cuatro especies saprófitas de los géneros Ttriphora (Orchidaceae) y Voyria (Gentianaceae) así como dos parásitas de los géneros $B d a-$ llophytum (Cytinaceae) y Phoradendron (Santalaceae). La RBSAT presenta una riqueza florística $(I B)$ de 79.53 especies por $\mathrm{km}^{2}$ la cual se encuentra entre los valores reportados para otras áreas de bosques tropicales estacionalmente secos

Tabla 1. Número de especies de la flora vascular registradas en la RBSAT Tanchipa, San Luis Potosí, México.

\begin{tabular}{lccc}
\hline Grupo Taxonómico & Familias & Géneros & Especies \\
\hline Helechos y afines & 4 & 7 & 11 \\
Gimnospermas & 1 & 2 & 2 \\
Angiospermas & 84 & 296 & 414 \\
Magnolídeas & 4 & 7 & 13 \\
Monocotiledóneas & 16 & 68 & 93 \\
Eudicotiledóneas & 64 & 221 & 308 \\
Total & 89 & 305 & 427 \\
\hline
\end{tabular}

Tabla 2. Familias de la flora vascular con mayor número de especies en la RBSAT, San Luis Potosí, México.

\begin{tabular}{lccc}
\hline Familias & Géneros & Especies & $\begin{array}{c}\text { \% del total } \\
\text { de especies }\end{array}$ \\
\hline Fabaceae & 28 & 40 & 9.37 \\
Orchidaceae & 23 & 28 & 6.56 \\
Poaceae & 15 & 21 & 4.92 \\
Asteraceae & 18 & 20 & 4.68 \\
Euphorbiaceae & 9 & 20 & 4.68 \\
Malvaceae & 14 & 19 & 4.45 \\
Apocynaceae & 12 & 13 & 3.04 \\
Convolvulaceae & 5 & 12 & 2.81 \\
Solanaceae & 4 & 12 & 2.81 \\
Acanthaceae & 6 & 11 & 2.58 \\
Rubiaceae & 7 & 11 & 2.58 \\
Bromeliaceae & 4 & 10 & 2.34 \\
Total & $145(47.54 \%)$ & $217(50.82 \%)$ & \\
\hline
\end{tabular}

en México (Tabla 4) que van de 47.73 a 168.47 especies por $\mathrm{km}^{2}$.

Endemismos y especies en riesgo. Se registraron 46 especies endémicas para México en 43 géneros y 22 familias, además de siete especies en alguna categoría de riesgo según la NOM-059-SEMARNAT-2010 (SEMARNAT 2010), (Apéndice 1).

Vegetación. La vegetación dominante dentro de RBSAT resultó ser la selva baja caducifolia que va de 300 a 600 $\mathrm{m}$ snm. Las especies dominantes en el estrato arbóreo son Beaucarnea inermis, Bursera simaruba, Guazuma ulmifolia, Karwinskia humboldtiana, Lysiloma divaricatum y Pseudobombax ellipticum. En la zona entre los 500 y $600 \mathrm{~m}$ snm, en sitios donde se forman cañadas y sumideros, existen selvas bajas subcaducifolias con dominancia de Beaucarnea inermis, Pseudobombax ellipticum, Robinsonella discolor, Harpalyce arborescens, Sapindus saponaria, Fraxinus dubia y Aphananthe monoica. En zonas por arriba de los $650 \mathrm{msnm}$

Tabla 3. Formas de crecimiento y tipos de nutrición especial (*) de las plantas vasculares de la RBSAT, San Luis Potosí, México.

\begin{tabular}{lcc}
\hline & No. especies & \% \\
\hline Hierba & 214 & 50.12 \\
Árbol & 78 & 18.27 \\
Arbusto & 59 & 13.82 \\
Trepadora & 47 & 11.01 \\
Epífita & 29 & 6.79 \\
Saprófita* & 4 & 0.94 \\
Parásita & 2 & 0.47 \\
\hline
\end{tabular}



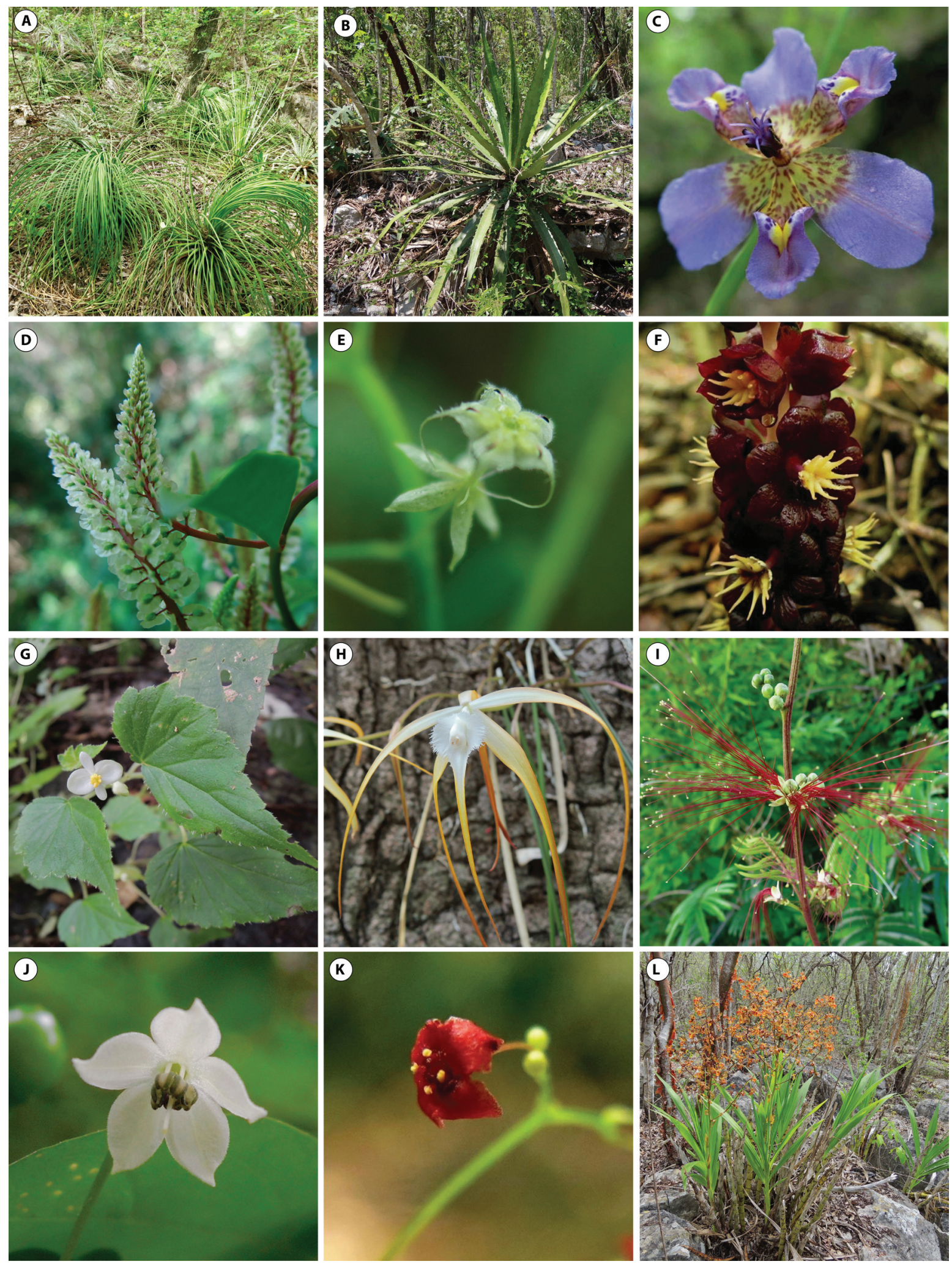

Figura 2. Algunas especies vegetales de la RBSAT. A) Agave tenuifolia; B) Agave univittata; C) Alophia drummondii; D) Anredera vesicaria; E) Ayenia pusilla; F) Bdallophytum americanum; G) Begonia gracilis; H) Brassavola cucullata; I) Calliandra houstoniana; J) Capsicum annuum var. glabriusculum; K) Crossopetalum uragoga; L) Cyrtopodium macrobulbon. Fotos: J.A. de Nova. 

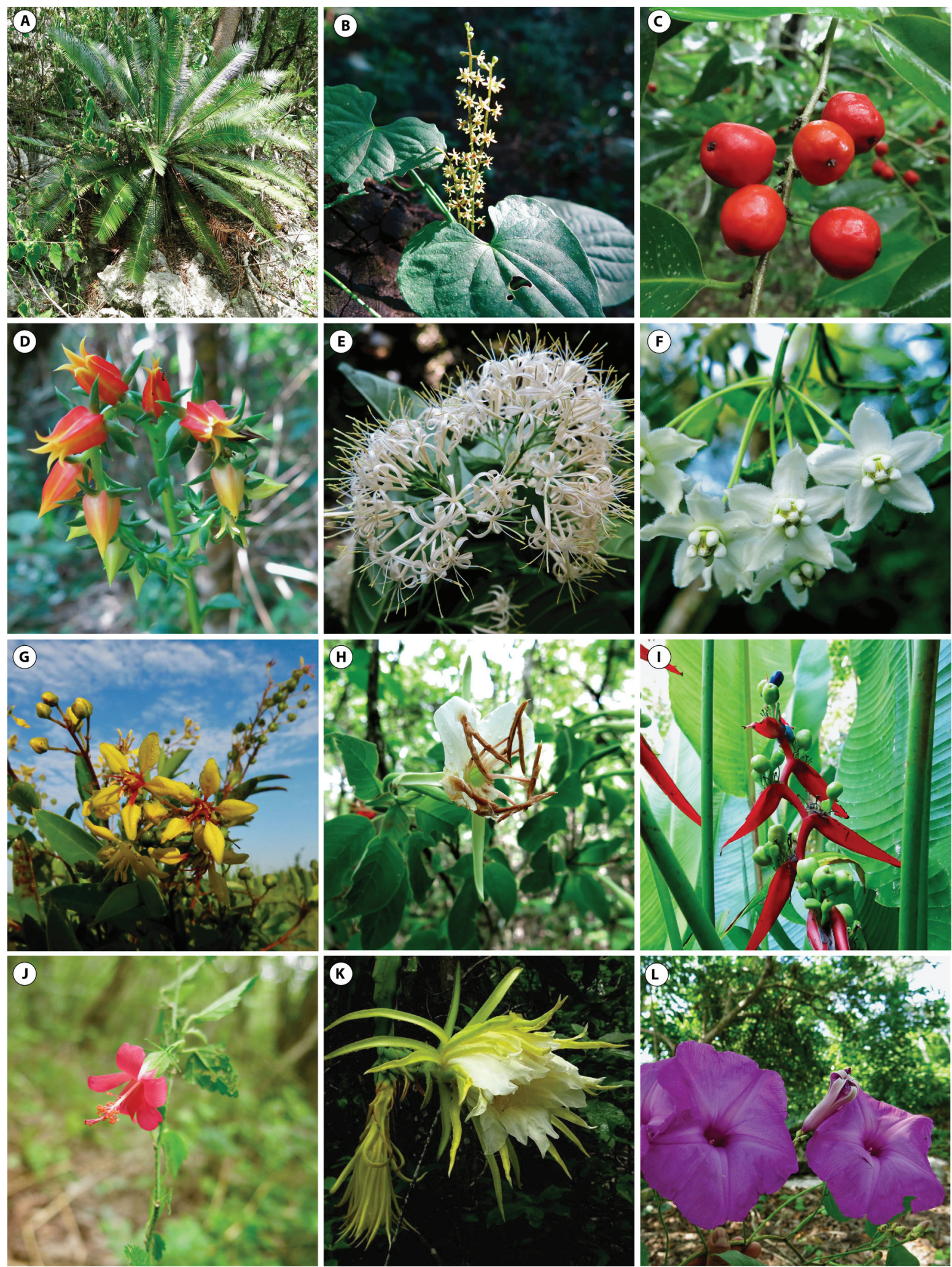

Figura 3. Algunas especies vegetales de la RBSAT. A) Dioon edule; B) Dioscorea pallens; C) Drypetes lateriflora; D) Echeveria tamaulipana; E) Exostema caribaeum; F) Funastrum pannosum; G) Galphimia glauca; H) Hauya elegans; I) Heliconia schiedeana; J) Hibiscus phoeniceus; K) Hylocereus undatus; L) Ipomoea squamosa. Fotos: J.A. de Nova. 

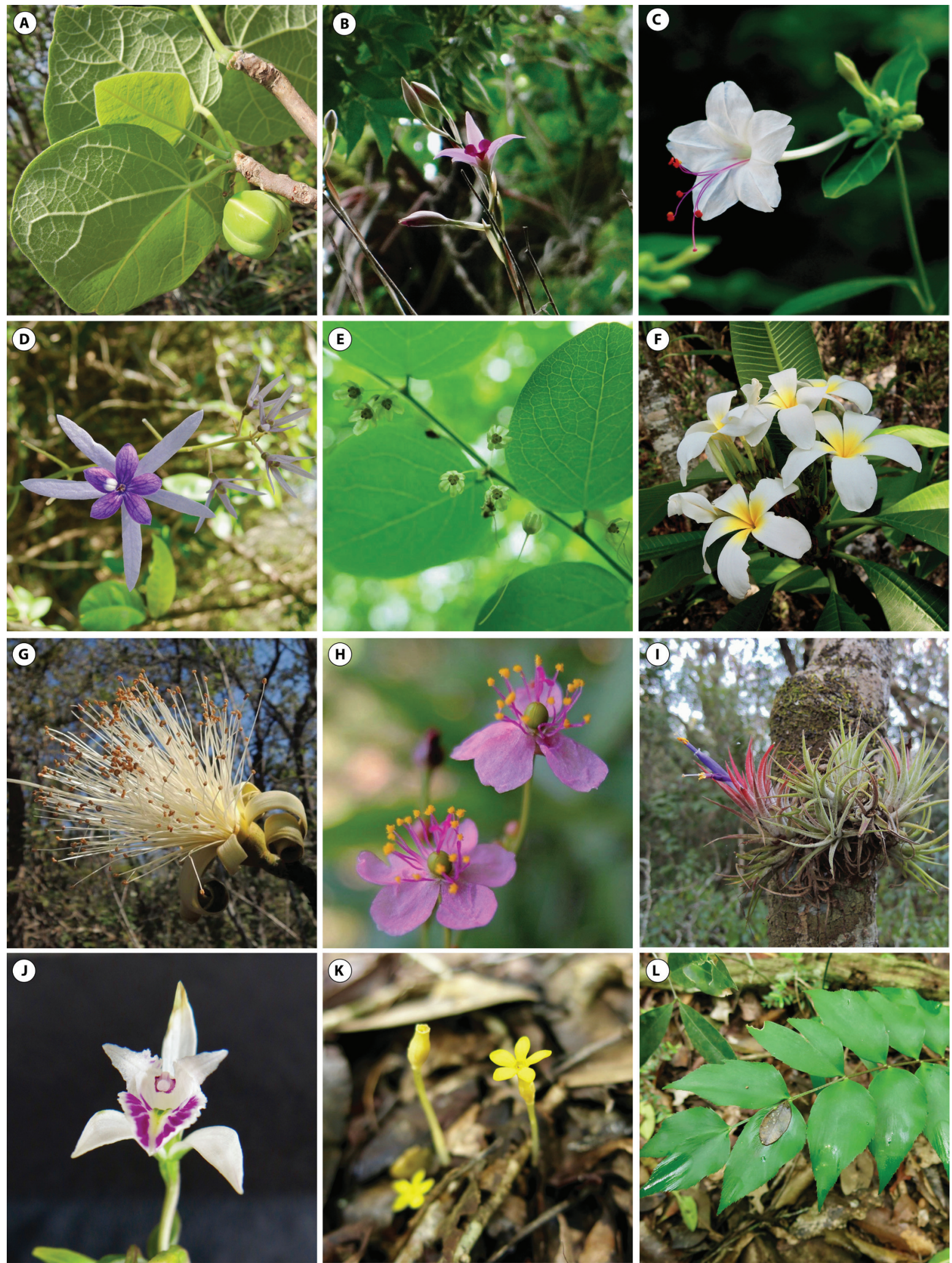

Figura 4. Algunas especies vegetales de la RBSAT. A) Jatropha sotoi-nunyezii B) Laelia anceps; C) Mirabilis longiflora; D) Petrea volubilis; E) Phyllanthus adenodiscus; F) Plumeria rubra; G) Pseudobombax ellipticum; H) Talinum paniculatum; I) Tillandsia ionantha; J) Triphora debilis; K) Voyria aphylla; L) Zamia fischeri. Foto B: L.E. Martínez Hernández. Fotos A, C, D, E, F, G, H, I, J, K, L: J.A. de Nova. 
De-Nova et al. / Botanical Sciences 97 (4): 761-788. 2019

Tabla 4. Índice de biodiversidad taxonómica para algunos bosques tropicales estacionalmente secos de México en comparación con la RBSAT.

\begin{tabular}{|c|c|c|c|c|}
\hline Estudio & Altitud & Área km² & no. spp. & IB sp/lnA \\
\hline Cañada La Chacona-Juan Crispín, Chiapas (Espinosa-Jiménez et al. 2014) & $594-1,030$ & 20.69 & 372 & 122.8 \\
\hline Chamela-Cuixmala, Jalisco (Lott \& Atkinson 2002) & $0-320$ & 131.42 & 551 & 113 \\
\hline Cuenca media-alta del río Actopan, Veracrúz (Castillo-Campos et al. 2007) & $400-900$ & 18.23 & 321 & 110.6 \\
\hline Cozumel, Q. Roo (Téllez-Valdés \& Cabrera-Cano 1987) & $0-10$ & 490 & 542 & 87.49 \\
\hline Venta Vieja, Gro. (Gual 1995) & $700-1,100$ & 38 & 307 & 84.39 \\
\hline Presente estudio. RBSAT, San Luis Potosí & $250-700$ & 214.64 & 427 & 79.53 \\
\hline Papalotepec, Gro. (Peralta 1995) & $700-1,350$ & 31 & 268 & 78.04 \\
\hline Tenabo, Campeche (Zamora-Crescencio 2003) & $1-100$ & 882 & 467 & 68.86 \\
\hline Bahía de Banderas-Puerto Vallarta, Jalisco-Nayarit (Ramírez-Delgadillo \& Cupul-Magaña 1999) & $0-2,000$ & 1,407 & 453 & 62.49 \\
\hline Gómez Farías, Tamaulipas (Valiente-Banuet et al. 1995) & $100-900$ & 400 & 286 & 47.73 \\
\hline
\end{tabular}

se desarrolla la selva mediana subcaducifolia con dominancia de Fraxinus dubia, Aphananthe monoica, Ficus spp., Robinsonella discolor, Sapindus saponaria y Annona globiflora. En la vertiente Este de la sierra, de 100 a 300 m snm, se encuentra una selva mediana subperenifolia con dominancia de Brosimum alicastrum, Aphananthe monoica, Lysiloma divaricatum y Bursera simaruba.

Usos. Los pobladores del área reconocen al menos 117 especies con algún uso o tóxicas y 153 nombres comunes para 136 especies (Apéndice 1), la categoría de uso con mayor riqueza de especies fue la de las plantas ornamentales (34), seguida por las especies usadas para construcción (33), como alimento (24) y medicinal (18). Otros usos registrados con menos frecuencia son leña (5), ebanistería (5), forraje (4), para la fabricación de utensilios de cocina (3), elaboración de herramientas de trabajo (Sida acuta) y finalmente como sustituto de jabón (Solanum erianthum). También son relevantes siete especies que los pobladores reconocen como tóxicas: Cascabela thevetia, Cnidoscolus multilobus, Croton ciliatoglandulifer, Croton cortesianus, Euphorbia schlechtendalii, Sebastiania pavoniana y Rhamnus humboldtiana. Las familias con un mayor número de especies usadas localmente fueron Orchidaceae (18 especies) principalmente como ornamentales y Fabaceae (14 especies) principalmente como material de construcción. Las especies con un mayor número de usos fueron Achatocarpus nigricans, Aphananthe monoica, Brahea dulcis y Quercus oleoides con tres usos cada una. Las formas de crecimiento que más usos tuvieron fueron arbustos (29 especies con nueve usos), árboles (41 especies con ocho usos), hierbas (24 especies con siete usos) y epífitas (16 especies con un uso).

\section{Discusión}

Riqueza florística. La RBSAT incluye al menos 427 especies de plantas superiores, lo que equivale aproximadamente a $13.24 \%$ (de 3,225 especies) o $9.49 \%$ (de 4,500 especies) de la diversidad de plantas vasculares del bosque tropical caducifolio o bosques estacionalmente secos de México, de acuerdo con Villaseñor (2016) y Rzedowski \& Calderón de Rzedowski (2013) respectivamente, y representan $9.16 \%$ (de 4,660 especies) de las plantas vasculares de los bosques neotropicales secos de América (Banda et al. 2016).

El índice de biodiversidad taxonómica $(I B=79.34$ $\mathrm{spp} . / \mathrm{km}^{2}$ ) dentro de la RBSAT es un poco menor al promedio estimado para otras regiones con bosques tropicales estacionalmente secos similares en México $\left(I B=85.46 \mathrm{spp} . / \mathrm{km}^{2}\right.$; Tabla 4) (Téllez-Valdés \& Cabrera-Cano 1987, Gual-Díaz 1995, Peralta 1995, Valiente-Banuet et al. 1995, RamírezDelgadillo \& Cupul-Magaña 1999, Lott \& Atkinson 2002, Zamora-Crescencio 2003, Castillo-Campos et al. 2007, Espinosa-Jiménez et al. 2014). Sin embargo, el rango varía de 47.73 a 122.77 , lo que sugiere cierta heterogeneidad taxonómica entre estas áreas. Pérez-García et al. (2012) han resaltado que los bosques tropicales secos incluyen una gran cantidad y variedad de tipos de vegetación, con diferencias substanciales en cuanto composición y estructura, lo que dificulta una comparación directa entre regiones.

Diversos autores han resaltado la importancia de las familias Apocynaceae, Asteraceae, Convolvulaceae, Euphorbiaceae, Fabaceae, Malvaceae, Orchidaceae y Poaceae para los bosques tropicales estacionalmente secos en diferentes regiones de México (Sánchez-Velásquez et al. 2002, White \& Hood 2004, Gallardo-Cruz et al. 2005, Lott \& Atkinson 2006, Alanís-Rodríguez et al. 2010, Pérez-García et al. 2012, Rzedowski \& Calderón de Rzedowski 2013, Dzib-Castillo et al. 2014), mismas que para la RBSAT agrupan el $40.38 \%$ de toda la riqueza específica. Particularmente la familia Fabaceae, que resulta ser la más rica en especies para el área estudiada, es también la mejor representada no sólo en los bosques secos de México (Rzedowski \& Calderón de Rzedowski 2013), sino también a lo largo del Neotrópico (Pennington et al. 2006). Otras familias ricas en especie que hasta hace poco fueron reconocidas como importantes para los bosques tropicales estacionales de México (Rzedowski \& Calderón de Rzedowski 2013) y que también resultan así para la RBSAT fueron Apocynaceae, Malvaceae y Orchidaceae. Además, algunos de los géneros con mayor riqueza específica en el área, como Euphorbia, Ipomoea y Tillandsia, han sido mencionados previamente entre los más ricos de los bosques tropicales caducifolios de México y en general 
para la flora del país (Rzedowski \& Calderón de Rzedowski 2013, Villaseñor 2016).

Formas de crecimiento. En los bosques tropicales estacionales la mayor riqueza de especies está constituida de manera variable por árboles, arbustos o hierbas (Gillespie et al. 2000, Trejo \& Dirzo 2002, Pérez-García et al. 2012). Para la RBSAT las hierbas fueron la forma de crecimiento con mayor número de especies, seguidas de los árboles, arbustos, trepadoras y epífitas, lo que coincide con otras áreas de bosques tropicales estacionalmente secos en México (ej. Espinosa-Jiménez et al. 2014, Castillo-Campos et al. 2007, Ramírez-Delgadillo \& Cupul-Magaña 1999). Resalta la presencia de especies con tipos de nutrición especial como son las parásitas y saprófitas, que hasta la fecha han sido poco reportadas en otros estudios para bosques tropicales estacionalmente secos.

Endemismos y especies en riesgo. Rzedowski \& Calderón de Rzedowski (2013) indican que el $7.9 \%$ de los géneros del bosque tropical caducifolio son endémicos de México, pero que su preponderancia varía mucho de un fragmento de bosque tropical a otro, y resalta que el endemismo se concentra en los bosques tropicales caducifolios de la vertiente pacífica, especialmente en la Cuenca del Balsas, e incluso existen bosques de la vertiente atlántica como los de Tamaulipas y Yucatán que aparentemente carecen de endemismos (Trejo \& Dirzo 2002). Sin embargo, la RBSAT incluye 46 especies endémicas de México, de 43 géneros y 22 familias, y destacan Orchidaceae, Euphorbiaceae y Asteraceae que suman el $44.6 \%$ del endemismo. Géneros que se consideran dominantemente mexicanos según Rzedowski \& Calderón de Rzedowski (2013), que se distribuyen principalmente en los bosques tropicales caducifolios y que estuvieron presentes en la RBSAT fueron Abutilon, Acalypha, Ayenia, Bursera, Caesalpinia, Calliandra, Cephalocereus, Colubrina, Cordia, Croton, Dioscorea, Euphorbia, Heliotropium, Ipomoea, Jatropha, Justicia, Leucaena, Lonchocarpus, Lysiloma, Manihot, Matelea, Mimosa, Passiflora, Randia, Ruellia, Senna, Serjania, Sida, Sideroxylon, Stenocereus, Tillandsia y Vachellia. En la RBSAT se registraron ocho especies con alguna categoría de conservación para la NOM-059-SEMARNAT 2010 (SEMARNAT 2010), las familias presentes fueron Arecaceae, Asparagaceae, Cactaceae, Fabaceae, Orchidaceae, Sapotaceae, y Zamiaceae. Cabe destacar que Zamiaceae y Arecaceae son grupos de plantas saqueadas intensamente con fines lucrativos (Quero 1994, Vovides \& Nicolalde-Morejón 2010). Otras familias importantes fueron Orchidaceae y Asparagaceae, pues de igual manera las orquídeas y las patas de elefante son extraídas de su hábitat, de manera ilegal, para ser vendidas como ornamentales (ContrerasHernández et al. 2008, Hernández-Sandoval et al. 2008).

Vegetación. En su trabajo sobre la vegetación de San Luis Potosí, Rzedowski (1965) describió al bosque tropical caducifolio que se localiza en lo que él denomina los declives orientales inferiores de la Sierra Madre Oriental entre San Martín Chalchicuautla y Ciudad Valles, región donde se lo- caliza la RBSAT. Este autor resalta la dominancia en el estrato arbóreo y arbustivo de especies como Guazuma ulmifolia, Lysiloma divaricatum, Harpalyce arborescens y Fraxinus dubia. Por otro lado, Puig (1991) dividió a los bosques tropicales de la región Huasteca en agrupaciones vegetales de acuerdo con las especies dominantes del estrato arbóreo y grupos ecológicos vinculados al tipo de suelo. A los bosques del norte de San Luis Potosí, como los de la RBSAT, los catalogó en la agrupación de Bursera simaruba y menciona que también puede incluir especies más características del bosque tropical subperennifolio como Dendropanax arboreus, Spondias mombin y Zuelania guidonia. Meave et al. (2012) mencionan que los bosques tropicales caducifolios que se desarrollan en áreas montañosas pueden albergar manchones de bosques con elementos pertenecientes a un matorral subtropical donde crecen especies espinosas de los géneros Parkinsonia, Lysiloma y Prosopis, y también es común encontrar áreas dominadas por palmares de Sabal mexicana o Brahea dulcis favorecidos por el disturbio humano, como ocurre en las regiones aledañas al área núcleo de la RBSAT.

Recientemente, De-Nova et al. (2018) describen cinco comunidades vegetales principales dentro de la RBSAT: selva baja espinosa caducifolia, selva baja caducifolia, selva baja subcaducifolia, selva mediana y selva mediana subperennifolia; las cuales varían en composición y estructura, siendo la más extensa la selva baja caducifolia. Otro tipo de vegetación que está presente de manera fragmentada y fuera del polígono de la reserva fue el encinar de Quercus oleoides. Los relictos de estos encinares han desaparecido casi en su totalidad en la región y actualmente sólo se encuentran unos pocos individuos aislados dentro de potreros y mezclados con vegetación secundaria fuera de las áreas naturales protegidas. La mayor parte de la vegetación de la reserva presenta etapas maduras de sucesión, lo que le confiere una marcada importancia en diversidad vegetal dentro de los ecosistemas presentes en la Huasteca Potosina si se considera que existe una proyección de estos tipos de vegetación hacia el norte (De-Nova et al. 2018).

Usos. Los pobladores de las comunidades cercanas a la reserva reconocen como útiles y/o tóxicas al menos 116 especies de plantas con 10 usos distintos. Estos datos superan las 55 especies útiles registradas previamente por Mora-Olivo et al. (1992). Los trabajos de etnobotánica que se han realizado en San Luis Potosí se centran en las comunidades indígenas y a menudo en ciertos usos como medicinal, comestible o construcción (Rzedowski 1967, Alcorn 1984, Cilia-López et al. 2015, Torres-Reyna et al. 2015). En el caso de la etnia Xi'uy, se reportan 27 especies usadas para la construcción (Torres-Reyna et al. 2015), mientras que en la RBSAT se registran 33. Para la zona de la etnia Teenek se registraron 54 especies comestibles en comunidades de Aquismón (CiliaLópez et al. 2015), mientras que en la RBSAT se registran 23 y en ambas regiones la familia con un mayor número de usos comestibles fue Fabaceae.

Implicaciones para la conservación. Algunas porciones de las comunidades vegetales cercanas a la RBSAT han experi- 
mentado cambios importantes desde la década de los setenta del siglo pasado, a raíz del proyecto gubernamental PujalCoy en la huasteca potosina cuyo objetivo fue transformar las "tierras ociosas" a productivas con actividades de agricultura y ganadería extensiva (Vargas 2010, Reyes-Hernández et al. 2018). Reyes-Hernández et al. (2006, 2018) realizaron estudios sobre los cambios de la cubierta vegetal del bosque tropical caducifolio y subcaducifolio de la llanura de San Luis Potosí del año 1973 al año 2000 y del año 1996 al 2016. En estos trabajos se indicó que en 1973 los bosques tropicales ocupaban alrededor de 98,270 hectáreas y en el 2000 alrededor de 10,216 hectáreas (Reyes-Hernández et al. 2006) lo que significó una tasa anual de deforestación del $11 \%$ similar a otras áreas con la misma vegetación, como Palenque en Chiapas y Tenosique en Tabasco. Sin embargo, estos autores indican que de 1996 al 2016 destaca la disminución de la transformación de las selvas que pasaron de 875 ha deforestadas en 1996 a sólo 175 ha en 2016 (Reyes-Hernández et al. 2018). Rubio-Méndez et al. (2018) indican que la RBSAT representa la región con el mejor estado de conservación, según datos demográficos, para las poblaciones de Beaucarnea inermis (pata de elefante), especie que ha sido afectada fuertemente por el saqueo en toda la región Huasteca. Otros estudios en el área son los de Ortega-Huerta \& Peterson (2004) quienes predicen a través de modelos, la importancia que tienen tres reservas de la biosfera del noreste de México para la protección de la distribución de aves y mamíferos en alguna categoría de riesgo; y encontraron que la RBSAT protege 113 especies de aves y 45 de mamíferos. OrtegaHuerta (2007) trabajó con la fragmentación del hábitat en varias reservas de la biosfera, incluyendo la RBSAT a la que le confirió valores altos en la prioridad de conservación para aves $(71 \%)$ y mamíferos $(23 \%)$ debido a sus bajos grados de fragmentación en comparación con otras reservas de la biosfera como la Sierra Gorda de Querétaro. Posteriormente Dueñas-López et al. (2013) mencionan a la RBSAT como una zona con altas probabilidades de servir como corredor ecológico para el jaguar debido a la presencia de bosques tropicales y pendientes moderadas, atributos más importantes hallados en este estudio para el hábitat del jaguar.

La riqueza de especies de plantas vasculares registradas para la RBSAT es notablemente alta en relación con su superficie y es similar a la registrada en otras regiones de bosque tropical estacionalmente seco. Los remanentes conservados de estos bosques en México y particularmente en el estado San Luis Potosí se encuentran bajo amenaza constante debido a que es una comunidad florística que se desarrolla solo en áreas con atributos muy particulares de suelo y clima, con una importante actividad humana agrícola y ganadera. En este sentido, el estado de conservación de las variantes de vegetación en la RBSAT que aquí se reportan, así como la presencia de endemismos y de especies en categoría de riesgo, resaltan su importancia para la conservación de los ecosistemas tropicales estacionalmente secos, y justifica su estatus cómo Área Natural Protegida, más aun sí se considera que existe una proyección de estos ecosistemas más hacia el norte representando un corredor ecológico entre los estados de San Luis Potosí y Tamaulipas.

\section{Agradecimientos}

Agradecemos al Consejo Nacional de Ciencia y Tecnología, a la Comisión Nacional para el Conocimiento y Uso de la Biodiversidad, la Secretaría de Educación Pública, la Comisión Nacional de Áreas Naturales Protegidas y SEMARNAT por el apoyo económico e infraestructura que permitió realizar esta investigación, particularmente a los proyectos CONACYT CB-2014/243454, CONABIO FB1829/PJ029/17, y SEP-PRODEP 103.5/13/6575 otorgados a JADNV. Al Ing. Alejandro Durán Fernández, director de la RBSAT, por todas las facilidades y el apoyo durante las investigaciones realizadas en la reserva. Agradecemos el apoyo en campo de Don Hermelindo Guzmán Antonia, Luis Enrique Martínez, Mercedes Elizabeth Ramírez Elías, Maywalida Montenegro Herrera. A Don José GarcíaPérez por la identificación y preparación de especímenes botánicos.

\section{Literatura citada}

Alanís-Rodríguez E, Aranda-Ramos RR, Mata-Balderas JM, Canizales-Velázquez PA, Jiménez-Pérez J, Uvalle-Sauceda JI, Valdecantos-Dema A, Ruiz-Bautista MG. 2010. Riqueza y diversidad de especies leñosas del bosque tropical caducifolio en San Luis Potosí, México. Ciencia UANL 13: $287-$ 293.

Alcorn JB. 1984. Huastec Mayan ethnobotany. Austin: University of Texas Press. ISBN-13: 978-0292715431

APG [Angiosperm Phylogeny Group], Chase MW, Christenhusz MJM, Fay MF, Byng JW, Judd WS, Soltis DE, Mabberley DJ, Sennikov AN, Soltis PS, Stevens PF. 2016. An update of the Angiosperm Phylogeny Group classification for the orders and families of flowering plants: APG IV. Botanical Journal of the Linnean Society 181: 1-20. DOI: https://doi.org/10.1111/boj.12385

Banda K, Delgado-Salinas A, Dexter KG, Linares-Palomino R, Oliveira-Filho A, Prado D. Weintritt J. et al.; DRYFLOR. 2016. Plant diversity patterns in neotropical dry forests and their conservation implications. Science 353: 1383-1387. DOI: https://doi.org/10.1126/science.aaf5080

Castillo-Campos G, Dávila-Aranda P, Zavala-Hurtado JA. 2007. La selva baja caducifolia en una corriente de lava volcánica en el centro de Veracruz: lista florística de la flora vascular. Boletín de la Sociedad Botánica de México 80: 77-104. DOI: https://doi.org/10.17129/botsci.1747

Cayuela L, Stein A, Oksanen J. 2017. Taxonstand: Taxonomic Standardization of Plant Species Names. https://CRAN.Rproject.org/package $=$ Taxonstand (accessed March 15, 2019)

Cervantes-Zamora Y, Cornejo-Olguín SL, Lucero-Márquez R, Espinoza-Rodríguez JM, Miranda-Víquez E, PinedaVelázquez A. 1990. Provincias Fisiográficas de México, Atlas Nacional de México. México: Instituto de Geografía, UNAM. http://conabio.gob.mx/informacion/metadata/gis/ rfisio4mgw.xml?_xsl=/db/metadata/xsl/fgdc_html.xsl\&_ indent $=$ no $($ accessed March 15, 2019)

Challenger A, Soberón J. 2008. Los ecosistemas terrestres. In: Sarukhán J, coord. Capital Natural de México, vol. I: Cono- 
cimiento Actual de la Biodiversidad. Comisión Nacional para el Conocimiento y Uso de la Biodiversidad. México. ISBN: 978-607-7607-03-8

Christenhusz M, Reveal J, Farjon A, Gardner MF, Mill RR, Chase MW. 2011a. A new classification and linear sequence of extant gymnosperms. Phytotaxa 19: 55-70.

DOI: http://dx.doi.org/10.11646/phytotaxa.19.1.3

Christenhusz M, Zhang XC, Schneider H. 2011b. A linear sequence of extant families and genera of lycophytes and ferns. Phytotaxa. 19: 7-54.

DOI: http://dx.doi.org/10.11646/phytotaxa.19.1.2

Cilia-López VG, Aradillas C, Díaz-Barriga F. 2015. Las plantas comestibles de una comunidad indígena de la Huasteca Potosina. Entreciencias 3: 143-152.

DOI: http://dx.doi.org/10.21933/J.EDSC.2015.07.144

CONABIO [Comisión Nacional para el Conocimiento y Uso de la Biodiversidad]. 2019. RB Sierra del Abra Tanchipa, San Luis Potosí. Portal Naturalista. $<$ https://www.naturalista. $\mathrm{mx} /$ projects/rb-sierra-del-abra-tanchipa-san-luis-potosi $>$ (accessed March 15, 2019).

CONANP [Comisión Nacional de Áreas Naturales Protegidas]. 2014. Programa de manejo. Reserva de la Biosfera Sierra del Abra Tanchipa. México: Secretaría de Medio Ambiente y Recursos Naturales/Comisión Nacional de Áreas Naturales Protegidas.

Contreras-Hernández A, Osorio-Rosales ML, Equihua-Zamora M, Benitez-Badillo G. 2008. Conservación y aprovechamiento de Beaucarnea recurvata, especie forestal no maderable. Cuadernos de Biodiversidad 28: 3-9.

DOI: http://dx.doi.org/10.14198/cdbio.2008.28.01

De-Nova JA, Medina R, Montero JC, Weeks A, Rosell JA, Olson ME, Eguiarte LE, Magallon S. 2012. Insights into the historical construction of species-rich Mesoamerican seasonally dry tropical forests: the diversification of Bursera (Burseraceae, Sapindales). New Phytologist 193: 276-287. DOI: https://doi.org/10.1111/j.1469-8137.2011.03909.x

De-Nova JA, Castillo-Lara P, Salinas-Rodríguez MM, Fortane1li-Martínez J, Mora-Olivo A. 2018. Los bosques tropicales estacionales. In: Reyes-Hernández H, De-Nova JA y Durán FA, eds. Reserva de la Biosfera Sierra del Abra Tanchipa. Biodiversidady Acciones para su Conservación. Universidad Autónoma de San Luis Potosí-Comisión Nacional de Áreas Natutales Protegidas-Fondo Mexicano para la Conservación de la Naturaleza-Universidad Autónoma de Tamaulipas. pp. 59-78. ISBN: 978-607-535-054-7

De-Nova JA, Sahagún-Sánchez FJ, Bueno-Villegas J, CruzadoCortes. 2019. Inventario multitaxonómico: PN El Potosí y RB Sierra del Abra Tanchipa (San Luis Potosí). Ciudad de México: Incidencia y Gobernanza Ambiental, A.C. Informe final SNIB- CONABIO, Proyecto No. PJ029.

Dick CW, Wright J. 2005. Tropical mountain cradles of dry forest diversity. Proceedings of the National Academy of Sciences 102: 10757-10758.

DOI: https://doi.org/10.1073/pnas.0505013102

DOF [Diario Oficial de la Federación].1994. Decreto por el que se declara como área natural protegida, con el carácter de reserva de la biosfera, la región conocida como Sierra del Abra Tanchipa, ubicada en los municipios de Ciudad Valles y Tamuín, Estado de San Luis Potosí. DF México. Lunes 6 de junio de 1994.

Dueñas-López G. 2013. Identificación de corredores biológicos potenciales para el jaguar (Panthera onca) en Sierra Abra Tanchipa, San Luis Potosi y sus limites estatales. MSc. Thesis. Colegio de Posgraduados.

Dzib-Castillo B, Chanatásig-Vaca C, González-Valdivia NA. 2014. Estructura y composición en dos comunidades arbóreas de la selva baja caducifolia y mediana subcaducifolia en Campeche, México. Revista Mexicana de Biodiversidad 85: 167-178. DOI: https://doi.org/10.7550/rmb.38706

Espinosa-Jiménez JA, López-Cruz A, Pérez-Farrera MA, López S. 2014. Inventario florístico de la cañada de La CachonaJuan Crispín y zonas adyacentes, Depresión Central de Chiapas, México. Botanical Sciences 92: 205-241. DOI: http://dx.doi.org/10.17129/botsci.30

Font Quer P. 1953. Diccionario de Botánica. España: Labor. ISBN: 84-8307-300-5

Gallardo-Cruz JA, Meave JA, García EAP. 2005. Estructura, composición y diversidad de la selva baja del Cerro Verde, Nizanda (Oaxaca), México. Boletín de la Sociedad Botánica de México 76: 19-35.

DOI: http://dx.doi.org/10.17129/botsci.1701

García E. 1981. Modificación al sistema de clasificación climática de Koëpen. México. D.F.: Instituto de Geografía, Universidad Nacional Autónoma de México. ISBN: 9703210104

Gual-Díaz M. 1995. Cañón del Zopilote (Área Venta Vieja). In: Diego-Pérez N, Fonseca R, eds. Estudios florísticos de Guerrero No. 6. México DF: Universidad Nacional Autónoma de México. ISBN: 968-36-4823-1

Gillespie TW, Grijalva A, Farris CN. 2000. Diversity, composition, and structure of tropical dry forests in Central America. Polish Journal of Ecology 147: 37-47.

DOI: https://doi.org/10.1023/A:1009848525399

Hernández-Sandoval L, Osorio-Rosales ML, Orellana-Lanza R, Martínez M, Pérez-Ferrera MA, Contreras-Hernández A, Malda-Barrera G, Espadas-Manrique C, Almanza-Rodríguez KE, Castillo-Gómez HA, Félix A. 2012. Manejo y conservación de las especies con valor comercial de Pata de elefante (Beaucarnea). Querétaro, México. Editorial Universitaria UAQ. 116 p.

INEGI [Instituto Nacional de Estadística y Geografía]. 1988. Hoja geológica (escala 1: 250 000). Aguascalientes, México. https://www.inegi.org.mx/temas/geologia/ (accessed January $15,2019)$.

INEGI. 2009. Guía para la interpretación de cartografía: uso del suelo y vegetación. Escala 1:250 000 Serie III. Aguascalientes, México. ISBN 978-607-494-015-2

INEGI. 2013. Conjunto de datos vectoriales edafológicos, escala 1:250000, serie II (continuo nacional). Aguascalientes, México.

Lott EJ, Atkinson TH. 2002. Biodiversidad y fitogeografía de Chamela-Cuitxmala, Jalisco. In: Nogera FA, Vega-Rivera JH, García-Aldrete AN, Quesada-Avendaño M, eds. Historia Natural de Chamela. Universidad Nacional Autónoma de México, México, D.F. ISBN: 9703205208,9789703205202

Lott EJ, Atkinson TH. 2006. Mexican and Central American seasonally dry tropical forests: Chamela-Cuixmala, Jalisco, as 
a focal point for comparison. In: Pennington RT, Lewis GP, Ratter JA. eds. Neotropical Savannas and Seasonally Dry Forests: Plant Diversity, Biogeography, and Conservation. Boca Raton: CRC Press, pp. 315-342.

DOI: https://doi.org/10.1201/9781420004496; ISBN-13: 978-0849329876

Meave JA, Romero-Romero MA, Salas-Morales SH, PérezGarcía EA, Gallardo-Cruz JA. 2012. Diversidad, amenazas y oportunidades para la conservación del bosque tropical caducifolio en el estado de Oaxaca, México. Revista Ecosistemas 21: 1-2.

Miranda F. Hernández-X E. 1963. Los tipos de vegetación de México y su clasificación. Boletín de la Sociedad Botánica de México 28: 29-179.

DOI: http://dx.doi.org/10.17129/botsci.1084

Mora-Olivo A, Sifuentes-Silva J, Margain-Hernández RM, Yáñez-Pacheco M. 1992. Recursos florísticos. In: Estudio de declaratoria como reserva ecológica de la Sierra del Abra Tanchipa, San Luis Potosí, México. Tamaulipas. México: Secretaría de Desarrollo Urbano y Ecología Delegación San Luis Potosí. Instituto de Ecología y Alimentos, Universidad Autónoma de Tamaulipas. pp. 79-105.

Ortega-Huerta MA. 2007. Fragmentation patterns and implications for biodiversity conservation in three biosphere reserves and surrounding regional environments, northeastern Mexico. Biological Conservation 134: 83-95.

DOI: https://doi.org/10.1016/j.biocon.2006.08.007

Ortega-Huerta MA, Peterson AT. 2004. Modelling spatial patterns of biodiversity for conservation prioritization in northeastern Mexico. Diversity and Distributions 10: 39-54. DOI: https://doi.org/10.1111/j.1472-4642.2004.00051.x

Pennington RT, Prado DE, Pendry CA. 2000. Neotropical seasonally dry forests and Quaternary vegetation changes. Journal of Biogeography 27: 261-273.

DOI: https://doi.org/10.1046/j.1365-2699.2000.00397.x

Pennington RT, Lavin M, Prado DE, Pendry CA, Pell S, Butterworth CA. 2004. Historical climate change and speciation: Neotropical seasonally dry forest plants show patterns of both Tertiary and Quaternary diversification. Philosophical Transactions of the Royal Society of London. Series B, Biological Sciences 359: 515-538.

DOI: https://doi.org/10.1098/rstb.2003.1435

Pennington RT, Ratter JA, Lewis GP, eds. 2006. Neotropical Savannas and Seasonally Dry Forests: Plant Biodiversity, Biogeography and Conservation. Boca Raton, Florida: CRC Press. ISBN 9780849329876

Pennington RT, Lavin M, Oliveira-Filho A. 2009. Woody plant diversity, evolution and ecology in the tropics: perspectives from seasonally dry tropical forests. The Annual Review of Ecology, Evolution, and Systematics 40: 437-457. DOI: https://doi.org/10.1146/annurev.ecolsys.110308.120327

Peralta S. 1995. Cañón del Zopilote (Papalotepec). In: Diego-Pérez N, Fonseca R, eds. Estudios florísticos de Guerrero No. 5. México, DF: Universidad Nacional Autónoma de México. ISBN: 968-36-4674-3

Pérez-García EA, Meave JA, Cevallos-Ferríz SR. 2012. Flora and vegetation of the seasonally dry tropics in Mexico: Origin and biogeographical implications. Acta Botánica
Mexicana 100: 149-193. http://www.redalyc.org/articulo. oa? id $=57424406007$

Pizano C, Cabrera M, García H. 2014. El bosque seco tropical en Colombia; generalidades y contexto. Bogotá, DC, Colombia: Instituto de Investigación de Recursos Biológicos Alexander von Humboldt (IAVH). ISBN: 9789588343976

ThePlantList. 2010. Version 1. Published on internet. http:// www.theplantlist.org/ (accessed March 15, 2019).

Puig H. 1991. Vegetación de la Huasteca (México), estudio fitogeográfico y ecológico. México. Instituto de Ecología AC, Institut Francais de Recherche Scientifique Pour le Developpement en Cooperation, entre de Etudes Mexicaines et Centramericaines.

Quero H. 1994. Las palmas de México: presente y futuro. Boletín de la Sociedad Botánica de México 55: 123-127. DOI: http://dx.doi.org/10.17129/botsci.1455

Ramírez-Delgadillo R, Cupul-Magaña FG. 1999. Contribución al conocimiento de la flora de Bahía de Banderas, NayaritJalisco, México. Ciencia Ergo Sum 6: 135-146.

Reyes-Hernández H, Aguilar-Robledo M, Aguirre-Rivera JR, Trejo-Vázquez I. 2006. Cambios en la cubierta vegetal y uso del suelo en el área del proyecto Pujal-Coy, San Luis Potosí, México, 1973-2000. Investigaciones geográficas 59: 26-42.

Reyes-Hernández H, Galarza-Rincón E, Vázquez-Villa BM. 2018. Dinámica de los cambios en la cubierta vegetal y usos de la tierra 1996-2016. In: Reyes-Hernández H., De-Nova JA, Durán FA, eds. Reserva de la Biosfera Sierra del Abra Tanchipa. Biodiversidad y Acciones para su Conservación. San Luis Potosí: UASLP-CONANP-RBSAT-FMCN-UAT. pp. 111-130. ISBN: 978607535 054-7

R Core Team. 2017. R, a language and environment for statistical computing. R Foundation for Statistical Computing, Vienna. https://www.R-project.org/ (accessed January 15, 2019).

Rubio-Méndez G, Castillo-Gómez HA, Hernández-Sandoval L, Espinosa-Reyes G, De-Nova JA. 2018. Chronic disturbance affects the demography and population structure of Beaucarnea inermis, a threatened species endemic to Mexico. Tropical Conservation Science 11: 1-12. DOI: https://doi.org/10.1177/1940082918779802

Rzedowski J. 1965. Vegetación en el Estado de San Luis Potosí. Acta Cientifica Potosina 5: 1-290.

Rzedowski J. 1967. Nombres regionales de algunas plantas de la Huasteca Potosina. Acta Científica Potosina 6: 7-58.

Rzedowski J. 1978. Vegetación de México. México, DF: Limusa.

Rzedowski J, Calderón de Rzedowski G. 1987. El bosque tropical caducifolio en la región mexicana del Bajío. Trace 12: 12-21.

Rzedowski J, Calderón de Rzedowski G. 2013. Datos para la apreciación de la flora fanerogámica del bosque tropical caducifolio de México. Acta Botánica Mexicana 102: 1-23.

Sánchez-Velásquez LR, Hernández-Vargas G, Carranza-M MA, Pineda-López MR, Cuevas-G R, Aragón-C F. 2002. Estructura arbórea del bosque tropical caducifolio usado para la ganadería extensiva en el norte de la Sierra de Manantlán, México. Antagonismo de usos. Polibotánica 13: 25-46.

SEMARNAT [Secretaría del Medio Ambiente y Recursos 
Naturales]. 2010. Norma Oficial Mexicana NOM-059-SEMARNAT-2010, Protección ambiental - Especies nativas de México de flora y fauna silvestres - Categorías de riesgo y especificaciones para su inclusión, exclusión o cambio - Lista de especies en riesgo. Diario Oficial de la Federación. 2da Sección, 30 de diciembre de 2010.

SLPM. 2019. Herbario Isidro Palacios, Universidaad Autónoma de San Luis Potosí. http://slpm.uaslp.mx/Antecedentes.aspx (accessed March 25, 2019)

Squeo FA, Cavieres LA, Arancio G, Novoa JE, Matthei O, Marticorena C, Rodríguez R, Arroyo TKM, Muñoz M. 1998. Biodiversidad de la flora vascular en la región de Antofagasta, Chile. Revista Chilena de Historia Natural 71: 571-591.

Téllez-Valdés O, Cabrera-Cano E. 1987. Listados florísticos de México VI. Flórula de la Isla de Cozumel, Q. R. México, DF: Universidad Nacional Autónoma de México. ISBN: 968-837-977-8.

Torres-Reyna JC, Fortanelli-Martínez J, van't Hooft A, Benitez-Gómez V. 2015. Etnobotánica de la vivienda rural en la región xi'iuy de la palma, San Luis Potosí, México. Etnobiología 13: 21-36.

Trejo-Vázquez I. 1999. El clima de la selva baja caducifolia en México. Investigaciones Geográficas 39: 40-52.

Trejo I, Dirzo R. 2002. Floristic diversity of Mexican seasonally dry tropical forests. Biodiversity and Conservation 11: 20482063. DOI: https://doi.org/10.1023/A:1020876316013

Valiente-Banuet A, González-Medrano F, Piñero D. 1995. La vegetación selvática de la región de Gómez Farías, Tamaulipas, México. Acta Botánica Mexicana 33: 1-36. DOI: https://doi.org/10.21829/abm33.1995.751

Vargas AV. 2010. La Reserva de la Biosfera Sierra del Abra Tanchipa: Las ANP de lo internacional a lo local. PhD Thesis, Universidad Autónoma de San Luis Potosí.

Villaseñor JL. 2016. Checklist of the native vascular plants of Mexico. Revista Mexicana de Biodiversidad 87: 559-902. DOI: https://doi.org/10.1016/j.rmb.2016.06.017

Vovides AP, Nicolalde-Morejón F. 2010. Fichas técnicas especies de cícadas mexicanas. En: Vovides AP, ed. Base de datos de las cícadas mexicanas. México, DF: Instituto de Ecología A.C. Bases de datos SNIB-CONABIO. Proyecto Núm. DK008.

White DA, Hood CS. 2004. Vegetation patterns and environmental gradients in tropical dry forests of the northern $\mathrm{Yu}-$ catan Peninsula. Journal of Vegetation Science 15: 151-160. DOI: https://doi.org/10.1111/j.1654-1103.2004.tb02250.x

Zamora-Crescencio P. 2003. Contribución al estudio florístico y descripción de la vegetación del municipio de Tenabo, Campeche, México. Polibotánica 15: 1-40.
Editor de sección: Martha González-Elizondo

Contribución de los autores: JADN (https://orcid.org/0000-00021989-1355) diseñó la investigación, realizó el trabajo de campo, la identificación taxonómica, el registro de los datos, su análisis, revisión de la nomenclatura y la estructuración del manuscrito. RGT (https://orcid.org/0000-0001-7295-8048) participó en la actualización de la información, la escritura y preparación de la versión final del manuscrito. PCL participó en el registro de los datos, la identificación taxonómica y en la estructuración del manuscrito. JFM (https://orcid. org/0000-0003-1958-7553) participó en el registro de los datos, la identificación taxonómica y en la estructuración del manuscrito. AMO (https://orcid.org/0000-0002-9654-0305) participó en el registro de los datos, la identificación taxonómica y en la estructuración del manuscrito. MMSR (https://orcid.org/0000-0001-7552-7809) participó en la actualización de la información, generación de mapas y tablas, y la escritura de versiones preliminares del manuscrito. Todos los autores han contribuido sustancialmente con ideas y revisiones de las versiones previas del manuscrito. 
Apéndice 1. Lista florística de la Reserva de la Biosfera Sierra de Abra Tanchipa. Recolectas depositadas en SLPM. Formas vitales: árbol (Ar); arbusto (Ab); Crassicaule (Cra), Epífita (Ep), Escandente (Es), Hierba (He), Palma (Pa), Parásita (Par), Herbácea saprófita (Sap) y Trepadora (Te). Usos: Alimento (A), Construcción (C), Ebanistería (E), Forraje (F), Herramientas $(\mathrm{H})$, Jabón (J), Leña (L), Medicinal (M), Ornamental (O), Postería (P), Tóxica (T), Utensilios (U). Categoría de riesgo (NOM059-SMARNAT-2010): amenazada (A), en peligro de extinción (P), en protección especial (Pr). Endémica de México: *. Recolectores: A.K. Cano Gudiño (AKCG); A. Mora-Olivo (AMO); B. Sobrevilla M. (BSM); C. Carranza A (CCA); F GómezLorence (FGL); F. Medellin Leal (FML); F. Takaki (FT); G. Martínez Calderón (GMC); G. Yatskievych (GY); H. GuzmánAntonia (HGA); J. Fortanelli Martínez (JFM); J. L. Mora-López (JLML); J. Rawlins (JR); J.A. de Nova Vazquez (JADV); J.B. Alcorn (JBA); J. Rzedowski R. (JRR); K. Hiramatsu (KH); L. Sabás R. (LSR); M.M. Salinas Rodríguez (MSR); P. Castillo-Lara (PCL); R. Padilla (RP); R. Puente (RPt); W.R. Anderson (WRA); Wm.W. Thomas (WWT).

\begin{tabular}{|c|c|c|c|c|c|c|}
\hline Clado/Familia/Especie & No. recolecta & Nombre común & $\begin{array}{c}\text { Forma } \\
\text { Vital }\end{array}$ & Usos & $\begin{array}{c}\text { Cat. } \\
\text { Riesgo }\end{array}$ & Endem. \\
\hline
\end{tabular}

\section{LYCOPHYA}

Selaginellaceae

Selaginella martensii Spring

PCL 868

Selaginella pallescens (C. Presl) Spring

\section{MONILOPHYTA}

Anemiaceae

Anemia adiantifolia (L.) Sw.

Observado

AMO 3429; JADNV 385;

MSR 114

Lygodium venustum $\mathrm{Sw}$.

\section{Polypodiaceae}

Microgramma nitida (J. Sm.) A.R. Sm.

Pleopeltis macrocarpa (Bory ex Willd.) Kaulf.

Pleopeltis polypodioides (L.) E.G. Andrews \& Windham

Polypodium longepinnulatum E. Fourn.

Polypodium loriceum L.

Polypodium plesiosorum Kunze

Polypodium polypodioides (L.) Watt

Pteridaceae

Adiantum tricholepis Fée

Cheilanthes aemula Maxon

Cheilanthes horridula Maxon

\section{GIMNOSPERMAE}

\section{Zamiaceae}

Dioon edule Lindl.

Zamia fischeri Miq. ex Lem.

\section{ANGIOSPERMAE}

MAGNOLIDAE

Aristolochiaceae

Aristolochia rhizantha Lundell

Annonaceae

Annona globiflora Schltdl.

AMO 3208, 3387

PCL 901

AMO 3213

Observado

Observado

AMO 3385

AMO 3214, 3385

AKGC 531

JADNV 382

MSR 21, 23, 38, 60, 69

AKGC 493
Observado

MSR 67; PCL 902

$\mathrm{He}$

$\mathrm{He}$

$\mathrm{He}$

$\mathrm{He}$

$\mathrm{He}$

$\mathrm{He}$

$\mathrm{He}$

$\mathrm{He}$

$\mathrm{He}$

$\mathrm{He}$

$\mathrm{He}$

$\mathrm{He}$
chamal, palma de dolores

chamalillo

$\mathrm{He}$

$\mathrm{He}$

$$
\begin{aligned}
& \mathrm{He} \\
& \mathrm{He}
\end{aligned}
$$

e

(1)

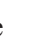

He


Flora de la Sierra del Abra Tanchipa

\begin{tabular}{|c|c|c|c|c|c|c|}
\hline Clado/Familia/Especie & No. recolecta & Nombre común & $\begin{array}{c}\text { Forma } \\
\text { Vital }\end{array}$ & Usos & $\begin{array}{c}\text { Cat. } \\
\text { Riesgo }\end{array}$ & Endem. \\
\hline \multicolumn{7}{|l|}{ Lauraceae } \\
\hline Cinnamomum tampicense (Meisn.) Kosterm. & JFM 699 & aguacatillo & $\mathrm{Ar}$ & & & * \\
\hline Ocotea tampicensis (Meisn.) Hemsl. & JADNV 259, 575 & aguacatillo & $\mathrm{Ar}$ & $\mathrm{C}$ & & \\
\hline Persea americana Mill. & JFM 723 & aguacate & $\mathrm{Ar}$ & $\mathrm{C}$ & & \\
\hline Persea schiedeana Nees & Observado & & $\mathrm{Ar}$ & & & \\
\hline \multicolumn{7}{|l|}{ Piperaceae } \\
\hline Peperomia blanda (Jacq.) Kunth & MSR 100 & & $\mathrm{He}$ & & & \\
\hline Peperomia glabella (Sw.) A.Dietr. & AMO 3384 & & $\mathrm{He}$ & & & \\
\hline Peperomia guatemalensis C.DC. ex Donn.Sm. & JLML 226 & & $\mathrm{He}$ & & & \\
\hline Peperomia liebmannii C. DC. & AKGC 456 & & $\mathrm{He}$ & & & \\
\hline Peperomia macrostachya (Vahl) A.Dietr. & PCL 890 & & $\mathrm{He}$ & & & \\
\hline Peperomia obtusifolia (L.) A.Dietr. & $\begin{array}{l}\text { AKGC 500; JFM 715; } \\
\text { JADNV 326; MSR } 101\end{array}$ & & $\mathrm{He}$ & & & \\
\hline Peperomia portulacifolia Kunth & JADNV 326 & & $\mathrm{He}$ & & & \\
\hline Piper amalago L. & JADNV 394 & yaxal, yaxil & $\mathrm{He}$ & M & & \\
\hline \multicolumn{7}{|l|}{ MONOCOTILEDÓNEAS } \\
\hline \multicolumn{7}{|l|}{ Alstroemeriaceae } \\
\hline Bomarea edulis (Tussac) Herb. & JADNV 645b; JFM 696 & & $\mathrm{He}$ & & & \\
\hline \multicolumn{7}{|l|}{ Amaryllidaceae } \\
\hline Zephyranthes chlorosolen (Herb.) D.Dietr. & JADNV 337, 355 & mayito & $\mathrm{He}$ & $\mathrm{O}$ & & \\
\hline Zephyranthes drummondii D.Don & AKGC 031 & & $\mathrm{He}$ & $\mathrm{O}$ & & \\
\hline \multicolumn{7}{|l|}{ Araceae } \\
\hline Arisaema macrospathum Benth. & $\begin{array}{l}\text { AMO 3386, 3395; JADNV } \\
370,633 ; \text { JFM } 720\end{array}$ & & $\mathrm{He}$ & & & \\
\hline Syngonium podophyllum Schott & MSR 116 & & $\mathrm{He}$ & & & \\
\hline \multicolumn{7}{|l|}{ Arecaceae } \\
\hline Acrocomia aculeata (Jacq.) Lodd. ex Mart. & Observado & coyol & $\mathrm{Pa}$ & & & \\
\hline Brahea dulcis (Kunth) Mart. & Observado & & $\mathrm{Pa}$ & $\begin{array}{l}\mathrm{C}, \mathrm{E}, \\
\mathrm{U}\end{array}$ & & \\
\hline Chamaedorea microspadix Burret & JADNV 368 & coralillo & $\mathrm{Pa}$ & & & \\
\hline Chamaedorea radicalis Mart. & PCL 897 & palmilla & $\mathrm{Pa}$ & $\mathrm{O}$ & & \\
\hline \multicolumn{7}{|l|}{ Asparagaceae } \\
\hline Agave tenuifolia Zamudio \& E. Sánchez & PCL 908 & & $\mathrm{He}$ & & & \\
\hline Agave univittata Haw. & $\begin{array}{l}\text { JADNV 372b; JFM 643; } \\
\text { PCL } 686\end{array}$ & lechuguilla & $\mathrm{He}$ & & & $*$ \\
\hline Beaucarnea inermis (S.Watson) Rose & $\begin{array}{l}\text { JADNV 534, 627; JFM 753; } \\
\text { PCL 957, } 958\end{array}$ & soyate & $\mathrm{Ar}$ & $\mathrm{O}$ & & $*$ \\
\hline $\begin{array}{l}\text { Echeandia chandleri (Greenm. \& C.H.Thomps.) } \\
\text { Cruden }\end{array}$ & AMO 3415 & & $\mathrm{He}$ & & & \\
\hline Manfreda maculosa (Hook.) Rose & PCL 2013-0809 & & $\mathrm{He}$ & & & \\
\hline Yucca treculeana Carrière & $\begin{array}{l}\text { JADNV s.n.; JRR 10200; } \\
\text { MSR } 56\end{array}$ & $\begin{array}{l}\text { palma datilera, } \\
\text { pita }\end{array}$ & $\mathrm{Pa}$ & A & & \\
\hline \multicolumn{7}{|l|}{ Bromeliaceae } \\
\hline Aechmea bracteata (Sw.) Griseb. & PCL 771,896 & & $\mathrm{He}$ & & & \\
\hline
\end{tabular}




\begin{tabular}{|c|c|c|c|c|c|c|}
\hline Clado/Familia/Especie & No. recolecta & Nombre común & $\begin{array}{c}\text { Forma } \\
\text { Vital }\end{array}$ & Usos & $\begin{array}{c}\text { Cat. } \\
\text { Riesgo }\end{array}$ & Endem. \\
\hline Bromelia pinguin $\mathrm{L}$. & $\begin{array}{l}\text { JRR 7473; PCL 685; JFM } \\
645\end{array}$ & huapilla & Ep & E, A & & \\
\hline Hechtia glomerata Zucc. & JRR 7474; PCL 687 & huapilla & $\mathrm{He}$ & & & \\
\hline Hechtia mexicana L.B.Sm. & Observado & & $\mathrm{He}$ & & $P$ & \\
\hline Tillandsia fasciculata $\mathrm{Sw}$. & JADNV 312 & & Ep & & & \\
\hline Tillandsia ionantha Planch. & $\begin{array}{l}\text { JADNV 287; JFM 737; PCL } \\
\text { s.n. }\end{array}$ & & Ep & & & \\
\hline Tillandsia juncea (Ruiz \& Pav.) Poir. & JADNV 887 & & Ep & & & \\
\hline Tillandsia recurvata (L.) L. & BSM s.n.; JRR 9736 & pastle & Ep & $\mathrm{O}$ & & \\
\hline Tillandsia schiedeana Steud. & $\begin{array}{l}\text { JFM 736; JADNV 590; PCL } \\
869 ; \text { MSR } 57\end{array}$ & gallito & Ep & & & \\
\hline Tillandsia usneoides (L.) L. & PCL 886 & pastle & Ep & $\mathrm{O}$ & & \\
\hline $\begin{array}{l}\text { Tillandsia utriculata subsp. pringlei (S.Watson) } \\
\text { C.S.Gardner }\end{array}$ & $\begin{array}{l}\text { JADNV } 367,598 ; \text { JFM 744; } \\
\text { PCL } 773,885\end{array}$ & & Ep & & & * \\
\hline \multicolumn{7}{|l|}{ Commelinaceae } \\
\hline Callisia repens (Jacq.) L. & MSR 103 & & $\mathrm{He}$ & & & \\
\hline Commelina erecta $\mathrm{L}$. & MSR 105 & & $\mathrm{He}$ & & & \\
\hline Commelina pallida Willd. & $\begin{array}{l}\text { JADNV } 295 ; \text { JFM 738; PCL } \\
853\end{array}$ & & $\mathrm{He}$ & & & \\
\hline Gibasis karwinskyana (Schult. \& Schult.f.) Rohweder & MSR 102 & & $\mathrm{He}$ & & & \\
\hline Gibasis pellucida (M.Martens \& Galeotti) D.R.Hunt & AMO 3383 & & $\mathrm{He}$ & & & \\
\hline Murdannia nudiflora (L.) Brenan & AMO 5082 & & $\mathrm{He}$ & & & \\
\hline Tradescantia zebrina Bosse & JADNV 379; PCL 864 & & $\mathrm{He}$ & & & \\
\hline \multicolumn{7}{|l|}{ Costaceae } \\
\hline Costus pulverulentus C.Presl & PCL 904 & caña de puerco & $\mathrm{He}$ & M & & \\
\hline \multicolumn{7}{|l|}{ Cyperaceae } \\
\hline Cyperus iria $\mathrm{L}$. & AMO 5085 & & $\mathrm{He}$ & & & \\
\hline Cyperus squarrosus $\mathrm{L}$. & AMO 5089 & & $\mathrm{He}$ & & A & \\
\hline Cyperus tenuis Sw. & JLML 224 & & $\mathrm{He}$ & & & \\
\hline Fimbristylis dichotoma (L.) Vahl & AMO 5083, 5084 & & $\mathrm{He}$ & & & \\
\hline Scleria gaertneri Raddi & $\begin{array}{l}\text { AKGC 452; JADNV 255, } \\
\text { 520; MSR 8; PCL } 860\end{array}$ & & $\mathrm{He}$ & & & \\
\hline Scleria lithosperma (L.) Sw. & AMO 3423 & & $\mathrm{He}$ & & & \\
\hline \multicolumn{7}{|l|}{ Dioscoreaceae } \\
\hline Dioscorea pallens Schltdl. & JADNV 521, 564; JFM 741 & & $\mathrm{Te}$ & & & \\
\hline \multicolumn{7}{|l|}{ Heliconiaceae } \\
\hline Heliconia schiedeana Klotzsch & Observado & papatla & $\mathrm{He}$ & & & \\
\hline \multicolumn{7}{|l|}{ Iridaceae } \\
\hline Alophia drummondii (Graham) R.C.Foster & PCL 862 & & $\mathrm{He}$ & $\mathrm{O}$ & A & \\
\hline Cipura campanulata Ravenna & AKGC 516 & & $\mathrm{He}$ & & & \\
\hline \multicolumn{7}{|l|}{ Marantaceae } \\
\hline Maranta arundinacea $\mathrm{L}$. & JADNV 525; PCL 873 & & $\mathrm{He}$ & & & \\
\hline \multicolumn{7}{|l|}{ Orchidaceae } \\
\hline Acianthera angustifolia (Lindl.) Luer & JADNV 587; JFM 807 & & Ep & $\mathrm{O}$ & & \\
\hline $\begin{array}{l}\text { Acianthera obscura (A.Rich. \& Galeotti) Pridgeon \& } \\
\text { M.W.Chase }\end{array}$ & PCL 879 & & Ep & & & $*$ \\
\hline
\end{tabular}


Flora de la Sierra del Abra Tanchipa

\begin{tabular}{|c|c|c|c|c|c|c|}
\hline Clado/Familia/Especie & No. recolecta & Nombre común & $\begin{array}{c}\text { Forma } \\
\text { Vital }\end{array}$ & Usos & $\begin{array}{l}\text { Cat. } \\
\text { Riesgo }\end{array}$ & Endem. \\
\hline Brassavola cucullata (L.) R.Br. & JADNV 325 & fantasmita & Ep & $\mathrm{O}$ & & \\
\hline Catasetum integerrimum Hook. & JADNV 334b ,549; PCL 772 & monjecito & Ep & $\mathrm{O}$ & & \\
\hline Cranichis sylvatica A.Rich. \& Galeotti & JADNV 577 & & Ep & $\mathrm{O}$ & & \\
\hline Cyclopogon cranichoides (Griseb.) Schltr. & Observado & & Ep & & & \\
\hline $\begin{array}{l}\text { Cyrtopodium macrobulbon (Lex.) G.A.Romero \& } \\
\text { Carnevali }\end{array}$ & JADNV 315 & & Ep & $\mathrm{O}$ & & \\
\hline Encyclia parviflora Regel & JADNV 322; JFM 649 & & Ep & $\mathrm{O}$ & & $*$ \\
\hline Epidendrum magnoliae Muhl. & JFM 804 & & Ep & & & \\
\hline Epidendrum raniferum Lindl. & JADNV 647; PCL 891 & & Ep & $\mathrm{O}$ & & \\
\hline Govenia alba A.Rich. \& Galeotti & JADNV 252, 535; PCL s.n. & lirio & $\mathrm{He}$ & $\mathrm{O}$ & & \\
\hline Habenaria novemfida Lindl. & JADNV 557; JFM 682 & lirio & $\mathrm{He}$ & $\mathrm{O}$ & & \\
\hline Isochilus unilateralis B.L.Rob. & PCL 872 & lirio & Ep & $\mathrm{O}$ & & $*$ \\
\hline Laelia anceps Lindl. & JADNV 360 & lirio & Ep & $\mathrm{O}$ & & $*$ \\
\hline Malaxis histionantha (Link) Garay \& Dunst. & JADNV 645, 648 & & $\mathrm{He}$ & & & \\
\hline Mesadenus lucayanus (Britton) Schltr. & Observado & & $\mathrm{He}$ & & & \\
\hline Nidema boothii (Lindl.) Schltr. & JFM s.n. & & Ep & $\mathrm{O}$ & & \\
\hline Notylia barkeri Lindl. & PCL 900 & & Ep & & & \\
\hline Oncidium ornithorhynchum Kunth & Observado & & Ep & & & \\
\hline Pelexia adnata (Sw.) Poit. ex Rich. & PCL 870, 871; JFM 826 & & $\mathrm{He}$ & & & \\
\hline Pelexia gutturosa (Rchb.f.) Garay & JADNV 254; PCL s.n. & & $\mathrm{He}$ & $\mathrm{O}$ & & \\
\hline Platythelys maculata (Hook.) Garay & JFM 793 & & $\mathrm{He}$ & & & \\
\hline Prosthechea cochleata (L.) W.E.Higgins & $\begin{array}{l}\text { JADNV 251, } 328 ; \text { JFM 704; } \\
\text { PCL s.n. }\end{array}$ & pulpito & Ep & $\mathrm{O}$ & & \\
\hline Prosthechea livida (Lindl.) W.E.Higgins & $\begin{array}{l}\text { JADNV 253, 316; JFM 754; } \\
\text { PCL } 889\end{array}$ & & Ep & $\mathrm{O}$ & & \\
\hline Sarcoglottis sceptrodes (Rchb.f.) Schltr. & JFM 670 & & $\mathrm{He}$ & & & \\
\hline Stanhopea tigrina Bateman ex Lindl. & JADNV 646, 650 & & Ep & & & \\
\hline $\begin{array}{l}\text { Trichocentrum biorbiculare (Balam \& Cetzal) } \\
\text { R.Jiménez \& Solano }\end{array}$ & PCL 774 & & Ep & $\mathrm{O}$ & & $*$ \\
\hline $\begin{array}{l}\text { Trichocentrum cosymbephorum (C.Morren) R.Jiménez } \\
\text { \& Carnevali }\end{array}$ & $\begin{array}{l}\text { AKGC } 460 ; \text { JADNV 300; } \\
\text { PCL } 909\end{array}$ & & Ep & $\mathrm{O}$ & & * \\
\hline Triphora debilis (Schltr.) Schltr. & $\begin{array}{l}\text { JADNV 591; JFM 717; PCL } \\
894\end{array}$ & & Sap & $\mathrm{O}$ & & \\
\hline \multicolumn{7}{|l|}{ Poaceae } \\
\hline Andropogon virginicus $\mathrm{L}$. & AMO 3380 & & $\mathrm{He}$ & & & \\
\hline Arundo donax $\mathrm{L}$. & Observado & carrizo & $\mathrm{He}$ & & & \\
\hline Bouteloua repens (Kunth) Scribn. \& Merr. & AMO 3419, 5086 & & $\mathrm{He}$ & F & & \\
\hline Brachiaria fasciculata (Sw.) Parodi & Observado & & $\mathrm{He}$ & & & \\
\hline Cenchrus echinatus L. & JADNV s.n. & & $\mathrm{He}$ & & & \\
\hline Digitaria insularis (L.) Mez ex Ekman & Observado & & $\mathrm{He}$ & & & \\
\hline Guadua velutina Londoño \& L.G.Clark & PCL 892 & otate & $\mathrm{He}$ & & & * \\
\hline Lasiacis divaricata (L.) Hitchc. & JFM 673 & zacate bambú & $\mathrm{He}$ & & & \\
\hline Lasiacis ruscifolia (Kunth) Hitchc. ex Chase & $\begin{array}{l}\text { JADNV 267; FT s.n.; MSR } \\
\text { 25; PCL s.n. }\end{array}$ & pacab, carricillo & $\mathrm{He}$ & & & \\
\hline Lasiacis sloanei (Griseb.) Hitchc. & AMO 3431 & & $\mathrm{He}$ & & & \\
\hline Leersia hexandra $\mathrm{Sw}$. & JFM 683 & & $\mathrm{He}$ & & & \\
\hline
\end{tabular}




\begin{tabular}{|c|c|c|c|c|c|c|}
\hline Clado/Familia/Especie & No. recolecta & Nombre común & $\begin{array}{c}\text { Forma } \\
\text { Vital }\end{array}$ & Usos & $\begin{array}{c}\text { Cat. } \\
\text { Riesgo }\end{array}$ & Endem. \\
\hline Leptochloa virgata (L.) P.Beauv. & AMO 5087 & & $\mathrm{He}$ & & & \\
\hline Oplismenus burmanni (Retz.) P.Beauv. & MSR 115 & & $\mathrm{He}$ & & & \\
\hline Panicum maximum Jacq. & $\begin{array}{l}\text { AMO 3381; FGL s.n.; JRR } \\
\text { 7470; RP s.n.; J. M. s.n. }\end{array}$ & zacate guinea & $\mathrm{He}$ & $\mathrm{F}$ & & \\
\hline Panicum miliaceum L. & MSR 84 & & $\mathrm{He}$ & & & \\
\hline Panicum trichoides Sw. & AMO 3428 & & $\mathrm{He}$ & & & \\
\hline Paspalum malacophyllum Trin. & AMO 3405 & & $\mathrm{He}$ & & & \\
\hline Paspalum mutabile Chase & AMO 3410 & & $\mathrm{He}$ & & & $*$ \\
\hline Paspalum plicatulum Michx. & AMO 3406 & & $\mathrm{He}$ & & & \\
\hline Schizachyrium tenerum Nees & AMO 5088 & & $\mathrm{He}$ & & & \\
\hline Setaria parviflora (Poir.) M.Kerguelen & AMO 3418 & & $\mathrm{He}$ & & & \\
\hline Trachypogon spicatus (L.f.) Kuntze & AMO 3437 & & $\mathrm{He}$ & & & \\
\hline Tripsacum dactyloides (L.) L. & AMO 3228, 5090 & & $\mathrm{He}$ & & & \\
\hline \multicolumn{7}{|l|}{ Smilacaceae } \\
\hline Smilax bona-nox $\mathrm{L}$. & JFM 716 & zarzaparrilla & $\mathrm{Te}$ & & & \\
\hline \multicolumn{7}{|l|}{ EUDICOTILEDÓNEAS } \\
\hline \multicolumn{7}{|l|}{ Acanthaceae } \\
\hline Elytraria bromoides Oerst. & JR 2811 & & $\mathrm{He}$ & & & \\
\hline Elytraria macrophylla Leonard & AKGC 506 & & $\mathrm{He}$ & & & \\
\hline Henrya insularis Nees & $\begin{array}{l}\text { JADNV 288, 291; MSR } \\
68,92\end{array}$ & & $\mathrm{He}$ & & & \\
\hline Justicia brandegeeana Wassh. \& L.B.Sm. & $\begin{array}{l}\text { AMO } 3215 ; \text { JADNV } 363 ; \\
\text { PCL } 852\end{array}$ & & $\mathrm{He}$ & & & \\
\hline Justicia leonardii Wassh. & AMO 3398 & & $\mathrm{He}$ & & & $*$ \\
\hline Justicia pilosella (Nees) Hilsenb. & $\begin{array}{l}\text { AKGC 430; JADNV 296, } \\
321,346,526 ; \text { JFM 739; } \\
\text { MSR 48, } 73\end{array}$ & & $\mathrm{He}$ & & & $*$ \\
\hline Justicia spicigera Schltdl. & $\begin{array}{l}\text { CCA s.n.; JADNV 323, 599; } \\
\text { MSR 11, 14, } 81\end{array}$ & mohuite, muu & $\mathrm{He}$ & & & \\
\hline Pseuderanthemum alatum (Nees) Radlk. & MSR 99 & & $\mathrm{He}$ & & & \\
\hline Ruellia nudiflora (Engelm. \& A.Gray) Urb. & JADNV 390 & & $\mathrm{He}$ & & & \\
\hline Ruellia paniculata $\mathrm{L}$. & MSR 89 & & $\mathrm{He}$ & & & \\
\hline Tetramerium nervosum Nees & $\begin{array}{l}\text { AMO 3394; JFM 639a; } \\
\text { MSR } 17\end{array}$ & & $\mathrm{He}$ & & & \\
\hline \multicolumn{7}{|l|}{ Achatocarpaceae } \\
\hline Achatocarpus nigricans Triana & JBA 2635; JFM 727 & $\begin{array}{l}\text { pimientillo, palo } \\
\text { dulce }\end{array}$ & $\mathrm{Ar}$ & $\begin{array}{l}\mathrm{F}, \mathrm{L} \\
\mathrm{P}\end{array}$ & & \\
\hline \multicolumn{7}{|l|}{ Amaranthaceae } \\
\hline Celosia nitida Vahl & $\begin{array}{l}\text { JADNV 546; AMO 3432; } \\
\text { MSR } 41\end{array}$ & & $\mathrm{He}$ & & & \\
\hline Iresine interrupta Benth. & KH s.n. & $\begin{array}{l}\text { chacaté, yerba } \\
\text { maiz }\end{array}$ & $\mathrm{Ab}$ & & & \\
\hline Iresine orientalis G.L. Nesom & MSR 1,86 & & $\mathrm{Ab}$ & & & $*$ \\
\hline \multicolumn{7}{|l|}{ Anacardiaceae } \\
\hline Spondias mombin $\mathrm{L}$. & Observado & $\begin{array}{l}\text { ciruela, jobo, } \\
\text { quínim }\end{array}$ & $\operatorname{Ar}$ & A & & \\
\hline
\end{tabular}




\begin{tabular}{|c|c|c|c|c|c|c|}
\hline Clado/Familia/Especie & No. recolecta & Nombre común & $\begin{array}{c}\text { Forma } \\
\text { Vital }\end{array}$ & Usos & $\begin{array}{c}\text { Cat. } \\
\text { Riesgo }\end{array}$ & Endem. \\
\hline \multicolumn{7}{|l|}{ Apocynaceae } \\
\hline Asclepias curassavica $\mathrm{L}$. & AKGC 542 & & $\mathrm{He}$ & & & \\
\hline Asclepias oenotheroides Schltdl. \& Cham. & JRR 10847 & & $\mathrm{He}$ & & & \\
\hline Cascabela thevetia (L.) Lippold & $\begin{array}{l}\text { JADNV 279, 365; JFM 761; } \\
\text { MSR 6; PCL s.n. }\end{array}$ & codo de fraile & $\mathrm{Ab}$ & $\mathrm{M}, \mathrm{T}$ & & \\
\hline Echites tuxtlensis Standl. & AKGC 444 & & Es & & & \\
\hline Funastrum pannosum (Hemsl.) Schltr. & Observado & & $\mathrm{He}$ & & & $*$ \\
\hline Marsdenia coulteri Hemsl. & JFM 650 & & $\mathrm{Te}$ & & & \\
\hline Matelea suberifera (B.L. Rob.) W.D. Stevens & Observado & & $\mathrm{Te}$ & & & $*$ \\
\hline $\begin{array}{l}\text { Pentalinon andrieuxii (Müll.Arg.) B.F.Hansen \& } \\
\text { Wunderlin }\end{array}$ & AKGC 518 & & $\mathrm{Te}$ & & & \\
\hline Plumeria rubra L. & JADNV 335c; PCL 777 & cacaloxuchitl & $\mathrm{Ar}$ & $\mathrm{O}$ & & \\
\hline Prestonia mexicana A.DC. & JFM 681 & & $\mathrm{Ar}$ & & & \\
\hline Rauvolfia tetraphylla $\mathrm{L}$. & JADNV 398; PCL 857 & & $\mathrm{He}$ & & & \\
\hline Tabernaemontana alba Mill. & WWT 2799 & & $\mathrm{Ab}$ & & & \\
\hline \multicolumn{7}{|l|}{ Basellaceae } \\
\hline Anredera vesicaria (Lam.) C.F.Gaertn. & JADNV 541 & zacacil & $\mathrm{He}$ & M & & \\
\hline \multicolumn{7}{|l|}{ Begoniaceae } \\
\hline Begonia gracilis Kunth & MSR 113 & & $\mathrm{He}$ & & & \\
\hline Begonia wallichiana Lehm. & AKGC 496 & & $\mathrm{He}$ & & & $*$ \\
\hline \multicolumn{7}{|l|}{ Bignoniaceae } \\
\hline Amphilophium crucigerum (L.) L.G.Lohmann & MSR 63 & & $\mathrm{Te}$ & & & \\
\hline Bignonia potosina (K.Schum. \& Loes.) L.G.Lohmann & JRR 7459 & & $\mathrm{Te}$ & & & \\
\hline Crescentia alata Kunth & JFM 672 & bexoste & $\mathrm{Ar}$ & & & \\
\hline Crescentia cujete L. & AKGC 543 & & Ar & & & \\
\hline Dolichandra unguis-cati (L.) L.G.Lohmann & MSR 7, 10 & & $\mathrm{Te}$ & & & \\
\hline Parmentiera aculeata (Kunth) Seem. & Observado & chote & Ar & $\mathrm{C}, \mathrm{F}$ & & \\
\hline Tabebuia rosea (Bertol.) Bertero ex A.DC. & Observado & palo de rosa & Ar & $\mathrm{C}$ & & \\
\hline Tecoma stans (L.) Juss. ex Kunth & JADNV 578 & tecuma & $\mathrm{Ab}$ & $\mathrm{C}$ & & \\
\hline \multicolumn{7}{|l|}{ Bixaceae } \\
\hline Amoreuxia wrightii A.Gray & JADNV 339 & & $\mathrm{He}$ & & & \\
\hline \multicolumn{7}{|l|}{ Boraginaceae } \\
\hline Cordia alba (Jacq.) Roem. \& Schult. & JRR 7765 & & $\mathrm{Ab}$ & & & \\
\hline Cordia alliodora (Ruiz \& Pav.) Oken & JFM s.n. & $\begin{array}{l}\text { palo tabaco, } \\
\text { trompillo }\end{array}$ & $\mathrm{Ab}$ & & & \\
\hline Cordia boissieri A.DC. & JRR 10201, 10408 & & $\mathrm{Ab}$ & & & \\
\hline Euploca fruticosa (L.) J.I.M.Melo \& Semir & AKGC 468 & & $\mathrm{He}$ & & & \\
\hline Nama dichotoma (Ruiz \& Pav.) Choisy & MSR 104 & & $\mathrm{He}$ & & & \\
\hline \multicolumn{7}{|l|}{ Burseraceae } \\
\hline Bursera fagaroides (Kunth) Engl. & JADNV 333 & copal & $\mathrm{Ar}$ & M & & \\
\hline Bursera simaruba (L.) Sarg. & JADNV 286 & chaca & Ar & M & & \\
\hline \multicolumn{7}{|l|}{ Cactaceae } \\
\hline Acanthocereus tetragonus (L.) Hummelinck & Observado & jacube & Cra & A & & \\
\hline Hylocereus undatus (Haw.) Britton \& Rose & $\begin{array}{l}\text { JADNV 393; JFM 735; PCL } \\
881,899\end{array}$ & pitajaya & Ep & A & & \\
\hline
\end{tabular}




\begin{tabular}{|c|c|c|c|c|c|c|}
\hline Clado/Familia/Especie & No. recolecta & Nombre común & $\begin{array}{c}\text { Forma } \\
\text { Vital }\end{array}$ & Usos & $\begin{array}{c}\text { Cat. } \\
\text { Riesgo }\end{array}$ & Endem. \\
\hline Mammillaria magnimamma Haw. & Observado & & Cra & & & $*$ \\
\hline Nopalea cochenillifera (L.) Salm-Dyck & Observado & nopal & Cra & A & & \\
\hline Nopalea dejecta (Salm-Dyck) Salm-Dyck & Observado & & $\mathrm{Cra}$ & A & & \\
\hline $\begin{array}{l}\text { Opuntia engelmannii subsp. lindheimeri (Engelm.) U. } \\
\text { Guzmán \& Mandujano }\end{array}$ & RPt 1616 & $\begin{array}{l}\text { cuija, oreja de } \\
\text { elefante }\end{array}$ & Cra & A & & \\
\hline Opuntia pubescens H.L.Wendl. ex Pfeiff. & AKGC 487; JADNV 320 & & Cra & A & & \\
\hline $\begin{array}{l}\text { Pilosocereus cometes (Scheidw.) Byles \& G.D. } \\
\text { Rowley }\end{array}$ & JFM 647 & & Cra & & $\operatorname{Pr}$ & $*$ \\
\hline Rhipsalis baccifera (J.S.Muell.) Stearn & $\begin{array}{l}\text { JADNV 271; MSR 59; PCL } \\
\text { s.n. }\end{array}$ & & Ep & & & \\
\hline Selenicereus spinulosus (DC.) Britton \& Rose & JADNV 334 & & Ep & & & \\
\hline \multicolumn{7}{|l|}{ Campanulaceae } \\
\hline Lobelia berlandieri A.DC. & MSR 78 & & $\mathrm{He}$ & & & \\
\hline \multicolumn{7}{|l|}{ Cannabaceae } \\
\hline Aphananthe monoica (Hemsl.) J.-F.Leroy & JADNV 361b; JFM 733 & ajuate, ajuatl & $\mathrm{Ar}$ & $\begin{array}{c}\mathrm{A}, \\
\mathrm{C}, \mathrm{E}, \\
\mathrm{P}\end{array}$ & & \\
\hline Celtis iguanaea (Jacq.) Sarg. & JRR 7446 & $\begin{array}{l}\text { granjeno, } \\
\text { huipuy }\end{array}$ & $\mathrm{Ar}$ & A & & \\
\hline \multicolumn{7}{|l|}{ Capparaceae } \\
\hline Forchhammeria sessilifolia Standl. & MSR 37 & pimiento & $\mathrm{Ar}$ & & & \\
\hline \multicolumn{7}{|l|}{ Caricaceae } \\
\hline Carica papaya $\mathrm{L}$. & MSR 80 & papaya & $\mathrm{Ab}$ & A & & \\
\hline \multicolumn{7}{|l|}{ Celastraceae } \\
\hline Crossopetalum uragoga (Jacq.) Kuntze & $\begin{array}{l}\text { AMO 3427; JADN 264; } \\
\text { MSR 15; PCL } 865\end{array}$ & & $\mathrm{He}$ & & & \\
\hline Hippocratea volubilis $\mathrm{L}$. & JFM 811 & & $\mathrm{Te}$ & & & \\
\hline Pristimera celastroides (Kunth) A.C.Sm. & MSR 13 & & $\mathrm{Te}$ & & & \\
\hline Wimmeria concolor Cham. \& Schltdl. & JADNV 643b, 643; JFM 824 & $\begin{array}{l}\text { algodoncillo, } \\
\text { escobillo }\end{array}$ & $\mathrm{Ar}$ & $\mathrm{P}$ & & \\
\hline \multicolumn{7}{|l|}{ Cleomaceae } \\
\hline Cleome aculeata $\mathrm{L}$. & AKGC 521 & & $\mathrm{He}$ & & & \\
\hline \multicolumn{7}{|l|}{ Compositae } \\
\hline Ageratina areolaris (DC.) Gage ex B.L.Turner & JADNV 260, 375; PCL s.n. & & $\mathrm{He}$ & & & \\
\hline Bidens pilosa $\mathrm{L}$. & $\begin{array}{l}\text { AMO3420; JADNV 543; } \\
\text { MSR 49, } 131\end{array}$ & & $\mathrm{He}$ & & & \\
\hline Brickellia diffusa (Vahl) A.Gray & MSR 125 & & $\mathrm{He}$ & & & \\
\hline Calea ternifolia Kunth & JRR 10388 & & $\mathrm{He}$ & & & \\
\hline Calea urticifolia (Mill.) DC. & MSR 118; PCL s.n. & & $\mathrm{He}$ & & & \\
\hline Chromolaena odorata (L.) R.M.King \& H.Rob. & JRR 10384; MSR 46 & & $\mathrm{He}$ & & & \\
\hline Florestina pedata (Cav.) Cass. & JRR 10367 & & $\mathrm{He}$ & & & \\
\hline Isocarpha oppositifolia (L.) Cass. & JRR 10371; MSR 9 & & $\mathrm{He}$ & & & \\
\hline Jefea lantanifolia (Schauer) Strother & AMO 3433; MSR 40 & & $\mathrm{He}$ & & & $*$ \\
\hline Melampodium gracile Less. & AMO 3403 & & $\mathrm{He}$ & & & \\
\hline Melampodium microcephalum Less. & JADNV 522 & & $\mathrm{He}$ & & & \\
\hline Montanoa tomentosa Cerv. & WRA 4062 & & $\mathrm{He}$ & & & \\
\hline
\end{tabular}




\begin{tabular}{|c|c|c|c|c|c|c|}
\hline Clado/Familia/Especie & No. recolecta & Nombre común & $\begin{array}{l}\text { Forma } \\
\text { Vital }\end{array}$ & Usos & $\begin{array}{c}\text { Cat. } \\
\text { Riesgo }\end{array}$ & Endem. \\
\hline Parthenium fruticosum Less. ex Schltdl. \& Cham. & AKGC 482; AMO 3433 & amargoso & $\mathrm{He}$ & & & $*$ \\
\hline $\begin{array}{l}\text { Porophyllum ruderale subsp. macrocephalum (DC.) } \\
\text { R.R.Johnson }\end{array}$ & JADNV 576 & pápalo quelite & $\mathrm{He}$ & A & & \\
\hline Schkuhria pinnata (Lam.) Kuntze ex Thell. & JADNV 354 & & $\mathrm{He}$ & & & \\
\hline $\begin{array}{l}\text { Sclerocarpus uniserialis (Hook.) Benth. \& Hook.f. ex } \\
\text { Hemsl. }\end{array}$ & JADNV 529; JRR 10382 & & $\mathrm{He}$ & & & \\
\hline Simsia eurylepis S.F.Blake & JRR 7440, 10387 & & $\mathrm{He}$ & & & $*$ \\
\hline Tithonia diversifolia (Hemsl.) A.Gray & PCL s.n. & & $\mathrm{He}$ & & & \\
\hline Trixis inula $\mathrm{Crantz}$ & MSR 120 & & $\mathrm{He}$ & & & \\
\hline Verbesina persicifolia DC. & JADNV 536, 568 & & $\mathrm{He}$ & & & \\
\hline \multicolumn{7}{|l|}{ Convolvulaceae } \\
\hline Evolvulus alsinoides (L.) L. & AMO 3417 & & $\mathrm{He}$ & & & \\
\hline Ipomoea batatas (L.) Lam. & JADNV 538 & camote & $\mathrm{Te}$ & & & \\
\hline Ipomoea hederifolia $\mathrm{L}$. & AMO 3389 & frijolillo & $\mathrm{Te}$ & & & \\
\hline Ipomoea jalapa (L.) Pursh & AMO 3396 & & $\mathrm{Te}$ & & & \\
\hline Ipomoea purga (Wender.) Hayne & AMO 3412 & & $\mathrm{Te}$ & & & \\
\hline Ipomoea reticulata O'Donell & AMO 3389 & & $\mathrm{Te}$ & & & \\
\hline Ipomoea squamosa Choisy & Observado & & $\mathrm{Te}$ & & & \\
\hline Ipomoea triloba $\mathrm{L}$. & AKGC 519 & & $\mathrm{Te}$ & & & \\
\hline Jacquemontia pentanthos (Jacq.) G. Don & AKGC 489 & & $\mathrm{Te}$ & & & \\
\hline Jacquemontia tamnifolia (L.) Griseb. & MSR 62 & & $\mathrm{Te}$ & & & \\
\hline Merremia dissecta (Jacq.) Hallier f. & JADNV 356 & & $\mathrm{Te}$ & & & \\
\hline Operculina pinnatifida (Kunth) O'Donell & $\begin{array}{l}\text { JADNV 341, 539; PCL 775; } \\
\text { JFM } 679\end{array}$ & & $\mathrm{Te}$ & & & \\
\hline \multicolumn{7}{|l|}{ Crassulaceae } \\
\hline $\begin{array}{l}\text { Echeveria tamaulipana Mart.-Aval., Mora-Olivo \& } \\
\text { M.Terry }\end{array}$ & JADNV 335b, 420; PCL 903 & & $\mathrm{He}$ & & & \\
\hline Echeveria walpoleana Rose & PCL 903 & & $\mathrm{He}$ & & & $*$ \\
\hline \multicolumn{7}{|l|}{ Cucurbitaceae } \\
\hline Melothria pendula $\mathrm{L}$. & MSR 85 & meloncito & $\mathrm{Te}$ & A & & \\
\hline \multicolumn{7}{|l|}{ Cytinaceae } \\
\hline Bdallophytum americanum (R.Br.) Eichler ex Solms & $\begin{array}{l}\text { JADNV 397; PCL 854; JFM } \\
730\end{array}$ & hongo & Par & & & \\
\hline \multicolumn{7}{|l|}{ Ebenaceae } \\
\hline Diospyros palmeri Eastw. & JADNV 632 & zapote negro & $\mathrm{Ar}$ & A & & $*$ \\
\hline \multicolumn{7}{|l|}{ Euphorbiaceae } \\
\hline Acalypha flavescens S.Watson & $\begin{array}{l}\text { JADNV } 314,336,369 \text {, } \\
374,395\end{array}$ & & $\mathrm{He}$ & & & \\
\hline Acalypha macrostachya Jacq. & JADNV 374 & & $\mathrm{He}$ & & & \\
\hline Adelia barbinervis Cham. \& Schltdl. & AKGC 616 & & $\mathrm{Ar}$ & & & $*$ \\
\hline Adelia oaxacana (Müll.Arg.) Hemsl. & JADNV 257, 317; PCL s.n. & & $\mathrm{Ar}$ & & & $*$ \\
\hline Bernardia albida Lundell & JADNV 329 & & $\mathrm{He}$ & & & $*$ \\
\hline Cnidoscolus multilobus (Pax) I.M.Johnst. & JFM 702 & mala mujer & $\mathrm{Ab}$ & $\mathrm{T}$ & & \\
\hline Croton argenteus $\mathrm{L}$. & AKGC 525 & & $\mathrm{He}$ & & & \\
\hline Croton ciliatoglandulifer Ortega & JADNV 399; JRR 10365 & solimán & $\mathrm{Ab}$ & $\mathrm{T}$ & & \\
\hline Croton cortesianus Kunth & JFM 633 & solimán & $\mathrm{Ab}$ & $\mathrm{T}$ & & \\
\hline
\end{tabular}




\begin{tabular}{|c|c|c|c|c|c|c|}
\hline Clado/Familia/Especie & No. recolecta & Nombre común & $\begin{array}{c}\text { Forma } \\
\text { Vital }\end{array}$ & Usos & $\begin{array}{l}\text { Cat. } \\
\text { Riesgo }\end{array}$ & Endem. \\
\hline Croton niveus Jacq. & MSR 74 & vara blanca & $\mathrm{Ab}$ & $\mathrm{P}$ & & \\
\hline Euphorbia colletioides Benth. & MSR 26 & & $\mathrm{Ab}$ & & & \\
\hline Euphorbia graminea Jacq. & AMO 3382 & & $\mathrm{He}$ & & & \\
\hline Euphorbia hypericifolia $\mathrm{L}$. & AKGC 478 & & $\mathrm{He}$ & & & \\
\hline Euphorbia pteroneura A.Berger & JADNV s.n. & & $\mathrm{He}$ & & & \\
\hline Euphorbia schlechtendalii Boiss. & JRR 10285,23345 & palo de leche & $\mathrm{He}$ & $\mathrm{T}$ & & \\
\hline Euphorbia tithymaloides L. & JADNV 280; PCL s.n. & $\begin{array}{l}\text { ácan-tele, } \\
\text { ácan-tzacam, } \\
\text { tzojol-tutub, }\end{array}$ & $\mathrm{He}$ & M & & \\
\hline Jatropha sotoi-nunyezii Fern.Casas \& E.Martínez & JADNV 345, 350; MSR 79 & piñón & $\mathrm{Ab}$ & $\mathrm{A}, \mathrm{L}$ & & $*$ \\
\hline Manihot pringlei S.Watson & JADNV 349 & & $\mathrm{He}$ & & & $*$ \\
\hline Sebastiania appendiculata (Müll.Arg.) Jabl. & AMO 3399 & & $\mathrm{Ab}$ & & & \\
\hline Sebastiania pavoniana (Müll.Arg.) Müll.Arg. & JADNV 263, 318 & palo de leche & $\mathrm{Ab}$ & $\mathrm{T}$ & & \\
\hline \multicolumn{7}{|l|}{ Fagaceae } \\
\hline Quercus oleoides Schltdl. \& Cham. & $\begin{array}{l}\text { JADNV 554, s.n.; JFM 630, } \\
\text { 631, 632; LSR } 314\end{array}$ & encino & $\mathrm{Ar}$ & $\begin{array}{l}\mathrm{C}, \mathrm{E} \\
\mathrm{L}, \mathrm{P}\end{array}$ & & \\
\hline \multicolumn{7}{|l|}{ Gentianaceae } \\
\hline Voyria flavescens Griseb. & JADNV 590b & & Sap & & & \\
\hline Voyria parasitica (Schltdl. \& Cham.) Ruyters \& Maas & MSR 58 & & Sap & & & \\
\hline Voyria tenella Guilding ex Hook. & JADNV 592b; PCL 1013 & & Sap & & & \\
\hline \multicolumn{7}{|l|}{ Lamiaceae } \\
\hline Callicarpa acuminata Kunth & $\begin{array}{l}\text { AMO 3207; JFM 709; MSR } \\
42\end{array}$ & & $\mathrm{He}$ & & & \\
\hline Ocimum campechianum Mill. & AMO 3233 & albacar loco & $\mathrm{He}$ & & & \\
\hline Ocimum carnosum (Spreng.) Link \& Otto ex Benth. & $\begin{array}{l}\text { AKGC 421; JFM 695; MSR } \\
72\end{array}$ & $\begin{array}{c}\text { albacar de } \\
\text { monte, albacar } \\
\text { loco }\end{array}$ & $\mathrm{He}$ & M & & \\
\hline Salvia coccinea Buc'hoz ex Etl. & JADNV 282; MSR 76 & & $\mathrm{He}$ & & & \\
\hline Salvia misella Kunth & MSR 109 & & $\mathrm{He}$ & & & \\
\hline Scutellaria seleriana Loes. & $\begin{array}{l}\text { JADNV 640; JFM 719; PCL } \\
866\end{array}$ & & $\mathrm{He}$ & & & \\
\hline Teucrium cubense Jacq. & JFM 677,686 & & $\mathrm{He}$ & & & \\
\hline \multicolumn{7}{|l|}{ Leguminosae } \\
\hline Acacia berlandieri Benth. & JRR 7455; MSR 52, 65 & & $\mathrm{Ar}$ & & & \\
\hline Acacia californica subsp. pringlei (Rose) L. Rico & JFM 823; MSR 3, 45, 112 & gavia huasteca & Ar & & & $*$ \\
\hline Acacia cornigera (L.) Willd. & AKGC 486 & & $\mathrm{Ar}$ & & & \\
\hline Aeschynomene fascicularis Cham. \& Schltdl. & MSR 2 & & $\mathrm{Te}$ & & & \\
\hline Bauhinia divaricata $\mathrm{L}$. & JFM 764 & pata de vaca & $\mathrm{Ab}$ & $\mathrm{O}$ & & \\
\hline Bauhinia macranthera Hemsl. & $\begin{array}{l}\text { JADNV 380; JFM 760; MSR } \\
50 ; \text { PCL 262, 330, 348, } 622\end{array}$ & & $\mathrm{Ab}$ & & & $*$ \\
\hline Caesalpinia mexicana A.Gray & MSR 121 & potro & $\mathrm{Ab}$ & $\mathrm{L}$ & & \\
\hline Caesalpinia pringlei (Britton \& Rose) Standl. & $\begin{array}{l}\text { JFM 641; MSR 122; PCL } \\
870\end{array}$ & & $\mathrm{Ab}$ & & & $*$ \\
\hline Calliandra houstoniana (Mill.) Standl. & $\begin{array}{l}\text { AMO3413; JADNV 630; } \\
\text { JRR } 7438\end{array}$ & & $\mathrm{Ab}$ & & & \\
\hline Canavalia septentrionalis J.D.Sauer & JADNV 639 & & $\mathrm{Te}$ & & & $*$ \\
\hline Centrosema sagittatum (Willd.) L.Riley & MSR 31 & & $\mathrm{Te}$ & & & \\
\hline
\end{tabular}




\begin{tabular}{|c|c|c|c|c|c|c|}
\hline Clado/Familia/Especie & No. recolecta & Nombre común & $\begin{array}{c}\text { Forma } \\
\text { Vital }\end{array}$ & Usos & $\begin{array}{c}\text { Cat. } \\
\text { Riesgo }\end{array}$ & Endem. \\
\hline Centrosema virginianum (L.) Benth. & JADNV 540 & & $\mathrm{Te}$ & & & \\
\hline Dalea scandens var. paucifolia (J.M.Coult.) Barneby & MSR 119 & hierba del burro & $\mathrm{He}$ & M & & \\
\hline Desmodium subsessile Schltdl. & AKGC 465 & & $\mathrm{He}$ & & & \\
\hline Diphysa microphylla Rydb. & JRR 10846, 10873 & & $\mathrm{Ab}$ & & & $*$ \\
\hline Ebenopsis ebano (Berland.) Barneby \& J.W.Grimes & JADNV 274; JFM 734 & ébano & $\mathrm{Ar}$ & $\mathrm{C}$ & & \\
\hline Enterolobium cyclocarpum (Jacq.) Griseb. & Observado & orejón & $\mathrm{Ar}$ & $\mathrm{C}, \mathrm{P}$ & & \\
\hline Erythrina americana Mill. & JFM 743 & & $\mathrm{Ar}$ & & & \\
\hline Erythrina nigrorosea (Krukoff \& Barneby) G.L.Nesom & $\begin{array}{l}\text { JADNV 347; PCL 882; MSR } \\
117\end{array}$ & colorín & $\mathrm{He}$ & & & \\
\hline Eysenhardtia polystachya (Ortega) Sarg. & JFM 642 & $\begin{array}{c}\text { palo azul, palo } \\
\text { dulce, vara } \\
\text { dulce }\end{array}$ & $\mathrm{Ab}$ & M & & \\
\hline Galactia striata (Jacq.) Urb. & AKGC 544; MSR 55 & & $\mathrm{Te}$ & & & \\
\hline Harpalyce arborescens A.Gray & $\begin{array}{l}\text { AKGC 437; JADNV 351, } \\
\text { 595; MSR } 123\end{array}$ & $\begin{array}{l}\text { chicharrillo, } \\
\text { chicharrilla }\end{array}$ & $\mathrm{Ar}$ & $\mathrm{C}$ & A & $*$ \\
\hline Havardia pallens (Benth.) Britton \& Rose & MSR 133 & $\begin{array}{l}\text { huacalero, } \\
\text { tenaza }\end{array}$ & $\mathrm{Ar}$ & $\mathrm{E}$ & & \\
\hline Indigofera miniata Ortega & AMO 3404 & & $\mathrm{He}$ & & & \\
\hline Leucaena leucocephala (Lam.) de Wit & JFM 701 & & $\mathrm{Ar}$ & & & \\
\hline Leucaena pulverulenta (Schltdl.) Benth. & AMO 3245,3436 & & $\mathrm{Ar}$ & & & \\
\hline Lonchocarpus rugosus Benth. & JFM 808; MSR 66 & & $\mathrm{Ar}$ & & & \\
\hline Lonchocarpus sp. nov. & JFM s.n. & & $\mathrm{Ar}$ & & & \\
\hline Lysiloma acapulcense (Kunth) Benth. & JFM s.n. & & $\mathrm{Ar}$ & $\mathrm{C}$ & & \\
\hline Lysiloma divaricatum (Jacq.) J.F.Macbr. & JFM 742 & rajador & $\mathrm{Ar}$ & $\mathrm{C}$ & & \\
\hline Lysiloma microphylla Benth. & JADNV 583 & rajador & $\mathrm{Ar}$ & $\mathrm{C}$ & & \\
\hline Mariosousa coulteri (Benth.) Seigler \& Ebinger & JRR 7445 & & $\mathrm{Ar}$ & & & \\
\hline Mucuna argyrophylla Standl. & Observado & & $\mathrm{Te}$ & & & \\
\hline Piscidia piscipula (L.) Sarg. & JADNV 332 & chijól & Ar & $\mathrm{C}, \mathrm{P}$ & & \\
\hline Pithecellobium dulce (Roxb.) Benth. & GMC 1859; JRR 7439 & guamúchil & $\mathrm{Ar}$ & & & \\
\hline Senegalia riparia (Kunth) Britton & MSR 75 & & $\mathrm{Te}$ & & & \\
\hline Senna atomaria (L.) H.S.Irwin \& Barneby & JADNV 281, 331; JFM 639b & $\begin{array}{l}\text { palo amarillo, } \\
\text { palo hediondo }\end{array}$ & $\mathrm{He}$ & M & & \\
\hline Senna lindheimeriana (Scheele) H.S.Irwin \& Barneby & MSR 77 & & $\mathrm{He}$ & & & \\
\hline Senna obtusifolia (L.) H.S.Irwin \& Barneby & AKGC 464 & & $\mathrm{He}$ & & & \\
\hline Vachellia cornigera (L.) Seigler \& Ebinger & JRR 7445; MSR 107, 132 & carnizuelo & $\mathrm{Ab}$ & $\mathrm{L}$ & & \\
\hline Vigna speciosa (Kunth) Verdc. & Observado & & $\mathrm{Te}$ & & & \\
\hline Zapoteca media (M.Martens \& Galeotti) H.M.Hern & JFM; MSR 32 & & $\mathrm{Te}$ & & & $*$ \\
\hline \multicolumn{7}{|l|}{ Lythraceae } \\
\hline Cuphea decandra Dryand. & AKGC 429 & & $\mathrm{He}$ & & & \\
\hline \multicolumn{7}{|l|}{ Malpighiaceae } \\
\hline Bunchosia lindeniana A.Juss. & AKGC 484 & & $\mathrm{Ar}$ & & & \\
\hline Callaeum septentrionale (A.Juss.) D.M.Johnson & JFM 755 & & $\mathrm{Ab}$ & & & $*$ \\
\hline Galphimia glauca Cav. & JADNV 373a, 516 & $\begin{array}{l}\text { árnica de raíz, } \\
\text { hierba del piojo }\end{array}$ & $\mathrm{Ab}$ & & & \\
\hline Gaudichaudia albida Schltdl. \& Cham. & JADNV 293; PCL 863 & & $\mathrm{Ab}$ & & & \\
\hline Gaudichaudia cynanchoides Kunth & JRR 7447 & & $\mathrm{Ab}$ & & & $*$ \\
\hline
\end{tabular}




\begin{tabular}{|c|c|c|c|c|c|c|}
\hline Clado/Familia/Especie & No. recolecta & Nombre común & $\begin{array}{c}\text { Forma } \\
\text { Vital }\end{array}$ & Usos & $\begin{array}{l}\text { Cat. } \\
\text { Riesgo }\end{array}$ & Endem. \\
\hline Gaudichaudia karwinskiana A.Juss. & AMO 3408 & & $\mathrm{Ab}$ & & & \\
\hline Heteropterys brachiata (L.) DC. & $\begin{array}{l}\text { JADNV 343; JFM 689; PCL } \\
\text { s.n. }\end{array}$ & & $\mathrm{Te}$ & & & \\
\hline Malpighia glabra L. & JADNV 558 & capulín & $\mathrm{Ab}$ & A & & \\
\hline \multicolumn{7}{|l|}{ Malvaceae } \\
\hline Abutilon trisulcatum (Jacq.) Urb. & JFM 646; JRR 7472 & chicleque & $\mathrm{He}$ & M & & \\
\hline Anoda cristata (L.) Schltdl. & AKGC 524 & & $\mathrm{He}$ & & & \\
\hline Anoda pedunculosa Hochr. & AKGC 490 & & $\mathrm{He}$ & & & $*$ \\
\hline Ayenia pusilla $\mathrm{L}$. & JRR 10389; MSR 18 & & $\mathrm{He}$ & & & \\
\hline Byttneria aculeata Jacq. & Observado & & $\mathrm{Te}$ & & & \\
\hline Ceiba pentandra (L.) Gaertn. & JADNV 600; PCL 877 & ceiba & $\mathrm{Ar}$ & $\mathrm{O}$ & & \\
\hline Gaya occidentalis (L.) Sweet & AKGC 529 & & $\mathrm{He}$ & & & \\
\hline Guazuma ulmifolia Lam. & AMO 3393; JFM s.n. & $\begin{array}{l}\text { aquiche, } \\
\text { guácima }\end{array}$ & $\mathrm{Ar}$ & M & & \\
\hline Hibiscus phoeniceus Jacq. & $\begin{array}{l}\text { AMO 3425; JADNV 589, } \\
\text { 591; MSR } 44\end{array}$ & & $\mathrm{He}$ & & & \\
\hline Melochia pyramidata $\mathrm{L}$. & JADNV 340 & & $\mathrm{He}$ & & & \\
\hline Melochia tomentosa $\mathrm{L}$. & AMO 3411 & & $\mathrm{He}$ & & & \\
\hline Phymosia umbellata (Cav.) Kearney & MSR 19 & & $\mathrm{He}$ & & & \\
\hline Pseudobombax ellipticum (Kunth) Dugand & $\begin{array}{l}\text { JADNV 278, 334a; JRR } \\
7456\end{array}$ & mocoque & $\operatorname{Ar}$ & $\mathrm{C}, \mathrm{O}$ & & \\
\hline Robinsonella discolor Rose \& Baker f. ex Rose & JFM 711; MSR 35 & palo balsa & $\mathrm{Ar}$ & & & \\
\hline Sida acuta Burm.f. & Observado & $\begin{array}{l}\text { malva, } \\
\text { malvarisco }\end{array}$ & $\mathrm{He}$ & $\mathrm{H}$ & & \\
\hline Sida elliottii Torr. \& A. Gray & AMO 3409 & & $\mathrm{He}$ & & & \\
\hline Sida linearis Cav. & AKGC 523 & & $\mathrm{He}$ & & & \\
\hline Sida rhombifolia $\mathrm{L}$. & MSR 128 & & $\mathrm{He}$ & & & \\
\hline Waltheria indica L. & JRR 7448 & & $\mathrm{He}$ & & & \\
\hline \multicolumn{7}{|l|}{ Meliaceae } \\
\hline Trichilia havanensis Jacq. & Observado & estribillo & $\mathrm{Ar}$ & $\mathrm{C}$ & & \\
\hline Trichilia hirta $\mathrm{L}$. & AKGC 509 & & $\mathrm{Ar}$ & & & \\
\hline \multicolumn{7}{|l|}{ Menispermaceae } \\
\hline Cissampelos pareira $\mathrm{L}$. & AKGC 520 & & $\mathrm{Te}$ & & & \\
\hline \multicolumn{7}{|l|}{ Moraceae } \\
\hline Brosimum alicastrum $\mathrm{Sw}$. & JADNV 381; JRR 7762 & ojite & $\mathrm{Ar}$ & $\mathrm{C}$ & & \\
\hline Castilla elastica Cerv. & AMO 3430 & hule & $\mathrm{Ar}$ & $\mathrm{U}$ & & \\
\hline Dorstenia drakena $\mathrm{L}$. & PCL 895 & & $\mathrm{He}$ & & & \\
\hline Ficus cotinifolia Kunth & JFM 692 & higuerón & $\mathrm{Ar}$ & $\mathrm{O}$ & & \\
\hline Ficus maxima Mill. & JFM 787 & copoy & Ar & & & \\
\hline Ficus pertusa L.f. & JADNV 637 & & $\mathrm{Ar}$ & & & \\
\hline Maclura tinctoria (L.) D.Don ex Steud. & JADNV 586; JFM 815 & mora & $\mathrm{Ar}$ & $\mathrm{C}$ & & \\
\hline Trophis racemosa (L.) Urb. & JFM 816 & & $\mathrm{Ar}$ & & & \\
\hline \multicolumn{7}{|l|}{ Myrtaceae } \\
\hline Calycorectes mexicanus O.Berg & Observado & & $\mathrm{Ab}$ & & & $*$ \\
\hline Eugenia capuli (Schltdl. \& Cham.) Hook. \& Arn. & $\begin{array}{l}\text { JADNV 644, t17-01; JFM } \\
\text { 706, 707; PCL } 893\end{array}$ & $\begin{array}{l}\text { capulín, } \\
\text { guayabilla }\end{array}$ & $\mathrm{Ab}$ & A & & \\
\hline
\end{tabular}




\begin{tabular}{|c|c|c|c|c|c|c|}
\hline Clado/Familia/Especie & No. recolecta & Nombre común & $\begin{array}{c}\text { Forma } \\
\text { Vital }\end{array}$ & Usos & $\begin{array}{c}\text { Cat. } \\
\text { Riesgo }\end{array}$ & Endem. \\
\hline Mosiera ehrenbergii (O.Berg) Landrum & MSR 5 & & $\mathrm{Ab}$ & & & $*$ \\
\hline Myrcianthes fragrans (Sw.) McVaugh & JFM 788 & guayabillo & $\mathrm{Ar}$ & $\mathrm{C}$ & & \\
\hline Psidium guajava $\mathrm{L}$. & Observado & guayaba & $\mathrm{Ar}$ & $\mathrm{A}, \mathrm{M}$ & & \\
\hline Psidium sartorianum (O.Berg) Nied. & $\begin{array}{l}\text { JADNV 266; JFM 750; PCL } \\
855\end{array}$ & guayabilla & $\mathrm{Ar}$ & $\mathrm{A}, \mathrm{M}$ & & \\
\hline \multicolumn{7}{|l|}{ Nyctaginaceae } \\
\hline Boerhavia erecta $\mathrm{L}$. & JADNV 400 & & $\mathrm{He}$ & & & \\
\hline Mirabilis jalapa $\mathrm{L}$. & JADNV 580 & maravilla & $\mathrm{He}$ & $\mathrm{O}$ & & \\
\hline Mirabilis longiflora $\mathrm{L}$. & JFM 810; MSR 28 & maravilla & $\mathrm{He}$ & & & \\
\hline Pisonia aculeata $\mathrm{L}$. & Observado & $\begin{array}{l}\text { chijól, loj, } \\
\text { nexcuahuitl }\end{array}$ & $\mathrm{Ar}$ & $\mathrm{P}$ & & \\
\hline \multicolumn{7}{|l|}{ Oleaceae } \\
\hline $\begin{array}{l}\text { Fraxinus dubia (Willd. ex Schult. \& Schult.f.) } \\
\text { P.S.Green \& M.Nee }\end{array}$ & $\begin{array}{l}\text { JADNV 268; JFM 710; PCL } \\
\text { s.n. }\end{array}$ & & $\mathrm{Ar}$ & & & \\
\hline \multicolumn{7}{|l|}{ Onagraceae } \\
\hline Hauya elegans DC. & PCL 960 & & $\mathrm{Ar}$ & & & \\
\hline \multicolumn{7}{|l|}{ Oxalidaceae } \\
\hline Oxalis corniculata $\mathrm{L}$. & Observado & trebolcillo & $\mathrm{He}$ & & & \\
\hline Oxalis decaphylla Kunth & AKGC 431 & & $\mathrm{He}$ & & & \\
\hline \multicolumn{7}{|l|}{ Passifloraceae } \\
\hline Passiflora coriacea Juss. & PCL 867 & & $\mathrm{Te}$ & & & \\
\hline Passiflora foetida $\mathrm{L}$. & AMO 3422; JRR 7441 & & $\mathrm{Te}$ & & & \\
\hline Passiflora serratifolia L. & MSR 47 & & $\mathrm{Te}$ & & & \\
\hline \multicolumn{7}{|l|}{ Phyllanthaceae } \\
\hline Margaritaria nobilis L.f. & PCL 857 & & $\mathrm{Ab}$ & & & \\
\hline Phyllanthus adenodiscus Müll.Arg. & $\begin{array}{l}\text { JADNV 283, 594; JFM 751; } \\
\text { PCL s.n. }\end{array}$ & & $\mathrm{He}$ & & & $*$ \\
\hline $\begin{array}{l}\text { Phyllanthus graveolens subsp. micrandrus (Müll.Arg.) } \\
\text { G.L.Webster }\end{array}$ & MSR 29; AKGC 449 & & $\mathrm{He}$ & & & \\
\hline Savia sessiliflora (Sw.) Willd. & MSR 24 & & $\mathrm{Ab}$ & & & \\
\hline \multicolumn{7}{|l|}{ Phytolaccaceae } \\
\hline Agdestis clematidea Moc. \& Sessé ex DC. & JRR 7437 & & $\mathrm{He}$ & & & \\
\hline Rivina humilis $\mathrm{L}$. & JADNV 292, 371; PCL 876 & & $\mathrm{He}$ & & & \\
\hline \multicolumn{7}{|l|}{ Plantaginaceae } \\
\hline Lophospermum erubescens D.Don & JFM s.n. & & $\mathrm{He}$ & & & \\
\hline Mecardonia procumbens (Mill.) Small & JFM 648 & $\begin{array}{l}\text { hierba de la } \\
\text { golondrina }\end{array}$ & $\mathrm{He}$ & M & & \\
\hline Russelia polyedra Zucc. & AMO 3226 & & $\mathrm{He}$ & & & \\
\hline \multicolumn{7}{|l|}{ Plumbaginaceae } \\
\hline Plumbago zeylanica $\mathrm{L}$. & MSR 90 & & $\mathrm{He}$ & & & \\
\hline \multicolumn{7}{|l|}{ Polygonaceae } \\
\hline Antigonon leptopus Hook. \& Arn. & JRR 7782 & & $\mathrm{He}$ & & & \\
\hline Coccoloba barbadensis Jacq. & $\begin{array}{l}\text { AMO 3230; JADNV 623, } \\
643 \mathrm{a}\end{array}$ & $\begin{array}{l}\text { pimientilla, } \\
\text { aguacatillo }\end{array}$ & $\mathrm{Ar}$ & & & \\
\hline Ruprechtia chiapensis Lundell & JRR 7465 & ajuate & $\mathrm{Ab}$ & & & \\
\hline
\end{tabular}




\begin{tabular}{|c|c|c|c|c|c|c|}
\hline Clado/Familia/Especie & No. recolecta & Nombre común & $\begin{array}{c}\text { Forma } \\
\text { Vital }\end{array}$ & Usos & $\begin{array}{c}\text { Cat. } \\
\text { Riesgo }\end{array}$ & Endem. \\
\hline Portulaca oleracea $\mathrm{L}$. & JADNV 545 & verdolaga & $\mathrm{He}$ & & & \\
\hline Portulaca pilosa $\mathrm{L}$. & JADNV 290; MSR 108 & verdolaga & $\mathrm{He}$ & A & & \\
\hline \multicolumn{7}{|l|}{ Primulaceae } \\
\hline Ardisia escallonioides Schltdl. \& Cham. & JFM 757 & & $\mathrm{Ab}$ & $\mathrm{M}, \mathrm{P}$ & & \\
\hline \multicolumn{7}{|l|}{ Putranjivaceae } \\
\hline Drypetes lateriflora (Sw.) Krug \& Urb. & $\begin{array}{l}\text { JADNV 517; JFM 714, 718, } \\
\text { 731; PCL 776, } 959\end{array}$ & palo café & $\mathrm{Ab}$ & $P$ & & \\
\hline \multicolumn{7}{|l|}{ Rhamnaceae } \\
\hline Colubrina elliptica (Sw.) Brizicky \& W.L.Stern & $\begin{array}{l}\text { JADNV 276, 616, 621; JFM } \\
638\end{array}$ & $\begin{array}{l}\text { palo amole, } \\
\text { guayacán, dat } \\
\text { zaat }\end{array}$ & $\mathrm{Ab}$ & $\mathrm{C}$ & & \\
\hline Colubrina greggii S.Watson & JFM s.n. & vara prieta & $\mathrm{Ab}$ & & & \\
\hline Krugiodendron ferreum (Vahl) Urb. & MSR 12 & hueso de tigre & $\mathrm{Ar}$ & & & \\
\hline Rhamnus humboldtiana Willd. ex Schult. & $\begin{array}{l}\text { FML 744; JADNV 284; JFM } \\
636\end{array}$ & $\begin{array}{c}\text { chalchanote, } \\
\text { tullidor, } \\
\text { sarabullo }\end{array}$ & $\mathrm{Ab}$ & $\mathrm{T}$ & & \\
\hline \multicolumn{7}{|l|}{ Rubiaceae } \\
\hline Bouvardia laevis M.Martens \& Galeotti & AMO 3390 & & $\mathrm{He}$ & & & \\
\hline Bouvardia multiflora (Cav.) Schult. & JADNV 593 & & $\mathrm{He}$ & & & \\
\hline Bouvardia ternifolia (Cav.) Schltdl. & JFM 789 & & $\mathrm{He}$ & & & \\
\hline Chiococca alba (L.) Hitchc. & $\begin{array}{l}\text { AMO } 3426 ; \text { JADNV 519; } \\
\text { PCL } 856\end{array}$ & & $\mathrm{Ab}$ & & & \\
\hline Chione venosa var. mexicana (Standl.) David W.Taylor & $\begin{array}{l}\text { JADNV 622, 634; JFM 726, } \\
814\end{array}$ & yerbaniz & $\mathrm{Ab}$ & $P$ & & \\
\hline Exostema caribaeum (Jacq.) Schult. & JFM 677,825 & clavelillo & $\mathrm{Ar}$ & $\mathrm{C}$ & & \\
\hline Exostema mexicanum A.Gray & JADNV 518, 566; JFM 651 & clavelillo & $\mathrm{Ar}$ & $\mathrm{C}$ & & \\
\hline Hamelia patens Jacq. & CCA s.n; JADNV 362,544 & & $\mathrm{He}$ & & & \\
\hline Psychotria erythrocarpa Schltdl. & JFM 634, 721; PCL 859 & & $\mathrm{Ab}$ & & & \\
\hline Randia laetevirens Standl. & JADNV 256, 319 & crucetillo & $\mathrm{Ab}$ & $\mathrm{U}$ & & \\
\hline Randia obcordata S.Watson & AKGC 428 & & $\mathrm{Ab}$ & & & \\
\hline \multicolumn{7}{|l|}{ Rutaceae } \\
\hline Esenbeckia berlandieri Baill. & JADNV 250; JFM 722 & hueso de tigre & $\mathrm{Ar}$ & $\mathrm{C}$ & & \\
\hline Esenbeckia runyonii C.V.Morton & AMO 3206 & limoncillo & $\mathrm{Ar}$ & & & \\
\hline Zanthoxylum fagara (L.) Sarg. & JFM 712 & uña de gato & $\mathrm{Ar}$ & & & \\
\hline \multicolumn{7}{|l|}{ Salicaceae } \\
\hline Neopringlea integrifolia (Hemsl.) S. Watson & AKGC 481 & & $\mathrm{Ar}$ & & & $*$ \\
\hline Xylosma flexuosa (Kunth) Hemsl. & JADNV 550; JFM 635 & & $\mathrm{He}$ & & & \\
\hline Zuelania guidonia (Sw.) Britton \& Millsp. & JADNV 273, 620; MSR 34 & volantín & $\mathrm{Ar}$ & $\mathrm{C}$ & & \\
\hline \multicolumn{7}{|l|}{ Santalaceae } \\
\hline Phoradendron brachystachyum (DC.) Nutt. & JFM 637 & $\begin{array}{c}\text { óclomte, } \\
\text { cuicilaca, } \\
\text { injerto, secapalo }\end{array}$ & Par & M & & \\
\hline \multicolumn{7}{|l|}{ Sapindaceae } \\
\hline Cardiospermum halicacabum $\mathrm{L}$. & JADNV 537, 573; MSR 87 & & $\mathrm{He}$ & & & \\
\hline Paullinia tomentosa Jacq. & JFM s.n.; PCL 694 & & $\mathrm{He}$ & & & \\
\hline Sapindus saponaria $\mathrm{L}$. & JFM 697; JRR 6956 & jaboncillo & $\mathrm{Ar}$ & & & \\
\hline
\end{tabular}


Flora de la Sierra del Abra Tanchipa

\begin{tabular}{|c|c|c|c|c|c|c|}
\hline Clado/Familia/Especie & No. recolecta & Nombre común & $\begin{array}{c}\text { Forma } \\
\text { Vital }\end{array}$ & Usos & $\begin{array}{c}\text { Cat. } \\
\text { Riesgo }\end{array}$ & Endem. \\
\hline Serjania racemosa Schumach. & JFM 713 & & $\mathrm{Te}$ & & & \\
\hline Urvillea ulmacea Kunth & AMO 3400; MSR 70 & & $\mathrm{Te}$ & & & \\
\hline \multicolumn{7}{|l|}{ Sapotaceae } \\
\hline Chrysophyllum mexicanum Brandegee & MSR 110 & & $\mathrm{Ar}$ & & & \\
\hline Sideroxylon capiri subsp. tempisque (Pittier) T.D.Penn. & JADNV 275, 313 & tempequiste & Ar & & A & \\
\hline \multicolumn{7}{|l|}{ Scrophulariaceae } \\
\hline Capraria frutescens (Mill.) Briq. & JADNV 294; MSR 83 & & $\mathrm{He}$ & & & \\
\hline \multicolumn{7}{|l|}{ Solanaceae } \\
\hline $\begin{array}{l}\text { Capsicum annuum var. glabriusculum (Dunal) Heiser } \\
\& \text { Pickersgill }\end{array}$ & JADNV 270; PCL s.n. & chile piquín & $\mathrm{He}$ & A & & \\
\hline Capsicum rhomboideum (Dunal) Kuntze & AKGC 434 & & $\mathrm{Ab}$ & & & \\
\hline Physalis gracilis Miers & AKGC 406 & & $\mathrm{He}$ & & & \\
\hline Physalis melanocystis (B.L.Rob.) Bitter & AKGC 411; JFM 724 & quiebra plato & $\mathrm{He}$ & & & \\
\hline Physalis pubescens $\mathrm{L}$. & JADNV 572 & & $\mathrm{He}$ & & & \\
\hline Solanum diphyllum $\mathrm{L}$. & AKGC 463 & & $\mathrm{Ab}$ & & & \\
\hline Solanum erianthum D. Don & MSR 126 & & $\mathrm{He}$ & $\mathrm{J}$ & & \\
\hline Solanum hirtum Vahl & $\begin{array}{l}\text { AKGC 514, 530; AMO } \\
3397 ; \text { GY 85-226 }\end{array}$ & & $\mathrm{He}$ & & & \\
\hline Solanum lanceifolium Jacq. & AKGC 537; JADNV 638 & & $\mathrm{He}$ & & & \\
\hline Solanum lycopersicum L. & Observado & tomate de monte & $\mathrm{He}$ & & & \\
\hline Solanum myriacanthum Dunal & GY 85-228 & & $\mathrm{He}$ & & & \\
\hline Solanum seaforthianum Andrews & HGA 17-01 & & $\mathrm{He}$ & & & \\
\hline Witheringia mexicana (B.L. Rob.) Hunz. & MSR 30 & & $\mathrm{He}$ & & & \\
\hline \multicolumn{7}{|l|}{ Talinaceae } \\
\hline Talinum paniculatum (Jacq.) Gaertn. & $\begin{array}{l}\text { JADNV 289, 377; PCL } 874, \\
888\end{array}$ & & $\mathrm{He}$ & & & \\
\hline \multicolumn{7}{|l|}{ Ulmaceae } \\
\hline \multicolumn{7}{|l|}{ Urticaceae } \\
\hline Pilea glabra S.Watson & $\begin{array}{l}\text { AMO 3388; JADNV 574; } \\
\text { JFM 762; JRR 7446; PCL } \\
778,875\end{array}$ & & $\mathrm{He}$ & & & \\
\hline Pilea microphylla (L.) Liebm. & AKGC 495 & & $\mathrm{He}$ & & & \\
\hline \multicolumn{7}{|l|}{ Verbenaceae } \\
\hline Citharexylum berlandieri S.Watson & $\begin{array}{l}\text { AMO 3246, 3391; JADNV } \\
542 ; \text { JFM 703; JRR } 10370\end{array}$ & & $\mathrm{Ar}$ & & & \\
\hline Lantana achyranthifolia Desf. & $\begin{array}{l}\text { AMO } 3189,3421 ; \text { MSR } \\
27,39\end{array}$ & & $\mathrm{He}$ & & & \\
\hline Lantana camara $\mathrm{L}$. & JFM 676 & & $\mathrm{He}$ & & & \\
\hline Lantana involucrata $\mathrm{L}$. & JRR 10380 & manzanita & $\mathrm{He}$ & & & \\
\hline Lippia alba (Mill.) N.E.Br. ex Britton \& P.Wilson & JADNV 324; JFM 680 & & $\mathrm{He}$ & & & \\
\hline Petrea volubilis $\mathrm{L}$. & JADNV 364 & $\begin{array}{l}\text { raspasombrero, } \\
\text { cacalacaxihuitl }\end{array}$ & $\mathrm{Te}$ & $\mathrm{O}$ & & \\
\hline Priva mexicana (L.) Pers. & AKGC 442; JADNV 401 & & $\mathrm{He}$ & & & \\
\hline Tamonea curassavica (L.) Pers. & AMO 3416 & & $\mathrm{He}$ & & & \\
\hline Verbena neomexicana (A.Gray) Briq. & JRR 7013 & & $\mathrm{He}$ & & & \\
\hline
\end{tabular}


De-Nova et al. / Botanical Sciences 97 (4): 761-788. 2019

\begin{tabular}{|c|c|c|c|c|c|c|}
\hline Clado/Familia/Especie & No. recolecta & Nombre común & $\begin{array}{c}\text { Forma } \\
\text { Vital }\end{array}$ & Usos & $\begin{array}{c}\text { Cat. } \\
\text { Riesgo }\end{array}$ & Endem. \\
\hline \multicolumn{7}{|l|}{ Violaceae } \\
\hline $\begin{array}{l}\text { Hybanthus attenuatus (Humb. \& Bonpl. ex Schult.) } \\
\text { Schulze-Menz }\end{array}$ & JFM 685; PCL 861 & $\begin{array}{l}\text { cuchara de } \\
\text { albañíl }\end{array}$ & $\mathrm{He}$ & & & \\
\hline Hybanthus mexicanus Ging. ex DC. & $\begin{array}{l}\text { AMO 3401; JADNV 625; } \\
\text { JRR } 14780\end{array}$ & & $\mathrm{He}$ & & & \\
\hline \multicolumn{7}{|l|}{ Vitaceae } \\
\hline Cissus microcarpa Vahl & MSR 61 & & $\mathrm{Te}$ & & & \\
\hline Cissus trifoliata (L.) L. & AMO 3219, 3249 & & $\mathrm{Te}$ & & & \\
\hline Vitis berlandieri Planch. & JADNV 327 & & $\mathrm{Te}$ & & & \\
\hline Vitis tiliifolia Humb. \& Bonpl. ex Schult. & AKGC 446 & & $\mathrm{Te}$ & & & \\
\hline
\end{tabular}

